\title{
BMJ Open Utility of models to predict 28-day or 30-day unplanned hospital readmissions: an updated systematic review
}

\author{
Huaqiong Zhou, ${ }^{1,2}$ Phillip R Della, ${ }^{2}$ Pamela Roberts, ${ }^{2}$ Louise Goh, ${ }^{2}$ \\ Satvinder S Dhaliwal ${ }^{2}$
}

To cite: Zhou H, Della PR, Roberts $P$, et al. Utility of models to predict 28-day or 30-day unplanned hospital readmissions: an updated systematic review. BMJ Open 2016;6: 011060 .

doi:10.1136/bmjopen-2016011060

\section{- Prepublication history and} additional material is available. To view please visit the journal (http://dx.doi.org/ 10.1136/bmjopen-2016011060).

Received 7 January 2016 Revised 17 May 2016 Accepted 23 May 2016

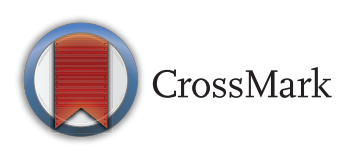

${ }^{1}$ Clinical Nurse, General Surgical Ward, Princess Margaret Hospital for Children, Perth, Western Australia, Australia ${ }^{2}$ School of Nursing, Midwifery and Paramedicine, Curtin University, Perth, Western Australia, Australia

Correspondence to Professor Satvinder Dhaliwal; s.dhaliwal@curtin.edu.au

\section{ABSTRACT}

Objective: To update previous systematic review of predictive models for 28-day or 30-day unplanned hospital readmissions.

Design: Systematic review.

Setting/data source: CINAHL, Embase, MEDLINE from 2011 to 2015.

Participants: All studies of 28-day and 30-day readmission predictive model.

Outcome measures: Characteristics of the included studies, performance of the identified predictive models and key predictive variables included in the models.

Results: $0 f 7310$ records, a total of 60 studies with 73 unique predictive models met the inclusion criteria. The utilisation outcome of the models included all-cause readmissions, cardiovascular disease including pneumonia, medical conditions, surgical conditions and mental health condition-related readmissions. Overall, a wide-range $\mathrm{C}$-statistic was reported in $56 / 60$ studies (0.21-0.88). 11 of 13 predictive models for medical condition-related readmissions were found to have consistent moderate discrimination ability (C-statistic $\geq 0.7$ ). Only two models were designed for the potentially preventable/avoidable readmissions and had $\mathrm{C}$-statistic $>0.8$. The variables 'comorbidities', 'length of stay' and 'previous admissions' were frequently cited across 73 models. The variables 'laboratory tests' and 'medication' had more weight in the models for cardiovascular disease and medical condition-related readmissions.

Conclusions: The predictive models which focused on general medical condition-related unplanned hospital readmissions reported moderate discriminative ability. Two models for potentially preventable/avoidable readmissions showed high discriminative ability. This updated systematic review, however, found inconsistent performance across the included unique 73 risk predictive models. It is critical to define clearly the utilisation outcomes and the type of accessible data source before the selection of the predictive model. Rigorous validation of the predictive models with moderate-to-high discriminative ability is essential, especially for the two models for the potentially preventable/avoidable readmissions. Given the limited available evidence, the development of a predictive

\section{Strengths and limitations of this study}

- This is an updated systematic review (20112015) of the literature relating to risk predictive models for unplanned hospital readmissions.

- This updated systematic review followed rigorous methodology applying comprehensive electronic database search, strict inclusion, exclusion and quality assessment criteria to synthesise current literature on characteristics and properties of risk predictive models for 28-day or 30-day unplanned hospital readmissions.

- The outcomes of the predictive models included in this systematic review were restricted to 28-day or 30-day unplanned hospital readmission.

model specifically for paediatric 28-day all-cause, unplanned hospital readmissions is a high priority.

\section{INTRODUCTION}

Unplanned hospital readmissions cause a disruption to the normality of patients and/or family/carers' lives and result in a significant financial burden on the healthcare system. ${ }^{12}$ In the USA, it has been estimated that 7.8 million $(20 \%)$ of hospital-discharged patients were readmitted. This accounted for $\$ 17.4$ billion of hospital payments by Medicare. ${ }^{3} 4$ In the UK, the figures suggested $\sim 35 \%$ of unplanned hospital readmissions, costing 11 billion pounds per annum (5.3 million admissions in 2010/2011). ${ }^{5}$

Unplanned hospital readmission rate is considered as a performance indicator to measure a hospital's quality of care. ${ }^{6}{ }^{7}$ Unplanned hospital readmission is defined as the percentage of unplanned or unexpected readmission to the same hospital within 28 days of being discharged. ${ }^{8} \quad 9$ However, the literature has widely used 
30 days within the context of measurement of hospital readmissions. ${ }^{167}$

One of the strategies to reduce the unplanned hospital readmission rate is the application of predictive models to identify patients at high risk for readmission. Preventive approaches can then be developed and applied to target the identified high-risk patients. A previous systematic review ${ }^{10}$ was conducted in 2011 on the risk predictive models for adult medical patients' hospital readmissions. A total of 30 studies with 26 predictive models were included, and the overall performance of reviewed models was poor. It is, however, worth noting that studies conducted in developing nations and studies that focused on paediatric patients and adult psychiatric and surgical patients were excluded.

Since 2011, there has been increased interest in either developing new predictive models or validating existing models due to high inpatient demand on the healthcare system. ${ }^{11-15}$ However, the performance of risk predictive models has varied significantly. The purpose of this systematic review is to update previous systematic review on predictive models for 28-day or 30-day unplanned hospital readmissions and to investigate and assess the characteristics of these models.

\section{METHODS}

\section{Search strategy and data sources}

An electronic database search was carried out using the CINAHL, Embase and MEDLINE to identify studies published between 2011 and 2015. The key search terms included 'unplanned readmission* or rehospitali*' AND ('predict*' AND 'model*') OR 'ROC or C-statistic*' OR 'sensitivity or specificity' (see online supplementary appendix 1 for full search strategy).

\section{Inclusion/exclusion criteria}

Articles eligible for inclusion were those published in English with full-text access from 2011 to 2015. Only peer-reviewed studies were included in this review. The study design of included studies needed to be clearly stated together with details of the performance of the risk predictive model reported. Abstract-only references were excluded. Studies included in the previous systematic review ${ }^{10}$ were excluded due to overlapping of the search period (1985-August 2011). Studies that included patients discharged from hospital but still receiving treatment, that is, intravenous antibiotics, via ambulatory care or hospital in the home programmes were also excluded.

\section{Study selection and data extraction}

Initial literature searches were conducted by $\mathrm{HZ}$ and PD. Two authors (HZ and LG) independently screened titles, abstracts and appraised full papers against the inclusion and exclusion criteria. The process of exclusion was relatively straightforward and only a handful of studies warranted discussion between the authors (HZ,
LG, SD, PD and PR) and to reach consensus as to whether they met the inclusion criteria.

Data were extracted from the final included studies by three authors (HZ, LG and SD). The data extraction included study characteristics, model performance and key variables of the predictive model. Study characteristics included study setting, population, data source, the timing of data collection, sample size, study design, model name if applicable, model utilisation outcome and readmission rate (table 1). Measures assessing predictive model performance, including discrimination, calibration, cut-off values used to identify patients at high risk of being readmitted to the hospital, sensitivity, specificity, positive predictive value (PPV) or negative predictive value (NPV), were extracted (table 2). Model discrimination is commonly assessed using C-statistic or the area under the receiver operating characteristic curve. Values of the C-statistic measurement range from 0.5 to 1.0 . A value of 0.5 indicates that the model is no better than chance at making a prediction of membership in a group, and a value of 1.0 indicates that the model perfectly identifies those within and not within a group. Models are typically considered reasonable when the C-statistic is higher than 0.7 and strong when the C-statistic exceeds $0.8 .^{71}$ Variables of the readmission risk predictive model were also extracted and presented in table 3. The studies were grouped based on the model utilisation outcome in the three tables. Disagreements between two reviewers about the extracted data were resolved through group discussion.

\section{Quality appraisal}

Six domains of potential bias ${ }^{72}$ were used to appraise the quality of included studies critically. The assessment of risk for bias was completed by two independent reviewers (HZ and SD). The ratings of 'yes', 'partly', 'no' or 'unsure' were given to each domain and then an overall risk of 'low' or 'high' was assigned to each study.

The six domains are:

1. Study participation: 'Was source population clearly defined?' and 'Was the study population described?' or 'Did the study population represent source population or population of interest?'

2. Study attrition: 'Was completeness of follow-up described and adequate?'

3. Prognostic factor measurement: 'Did prognostic factors measure appropriately?'

4. Outcome measurement: 'Was outcome defined and measured appropriately?'

5. Confounding measurement and account: 'Were confounders defined and measured?'

6. Analysis: 'Was analysis described and appropriate?' and 'Did analysis provide sufficient presentation of data?'

\section{Data synthesis}

Pooling of quantitative data was not possible as the included studies were not homogeneous. Therefore, the 
Table 1 Characteristics of 49 included studies on 28-day or 30-day unplanned hospital readmission (UHR) predictive models

\begin{tabular}{|c|c|c|c|c|c|c|c|}
\hline Reference & Model name & Model outcome & $\begin{array}{l}\text { Study design/data } \\
\text { source }\end{array}$ & Sample size & Age group (years) & $\begin{array}{l}\text { Duration of } \\
\text { retrieved data } \\
\text { source }\end{array}$ & $\begin{array}{l}\text { Readmission } \\
\text { rate }\end{array}$ \\
\hline \multicolumn{8}{|l|}{ All-cause UHRs (14) } \\
\hline $\begin{array}{l}\text { Escobar et } a l^{16} \\
\text { USA }\end{array}$ & $\begin{array}{l}\text { ED } 30 \\
\text { Discharge } 30 \text { LACE } \\
\text { (validation) }\end{array}$ & $\begin{array}{l}\text { 30-day all-cause } \\
\text { readmissions }\end{array}$ & $\begin{array}{l}\text { Retrospective cohort } \\
21 \text { hospitals } \\
\text { Electronic medical } \\
\text { records }\end{array}$ & $\begin{array}{l}\text { A total of } 360036 \\
\text { patients } \\
179978 \text { derivation set } \\
180058 \text { validation set }\end{array}$ & Mean $=64.1$ & $\begin{array}{l}1 \text { June 2010- } \\
31 \text { December } \\
2013\end{array}$ & $\begin{array}{l}\text { Derivation: } \\
\text { 12.5\%; Validation: } \\
12.4 \%\end{array}$ \\
\hline $\begin{array}{l}\text { Yu et al }{ }^{17} \\
\text { USA }\end{array}$ & $\begin{array}{l}\text { Institution-specific prediction } \\
\text { model } \\
\text { LACE (validation) }\end{array}$ & $\begin{array}{l}\text { 30-day all-cause } \\
\text { readmission }\end{array}$ & $\begin{array}{l}\text { Retrospective cohort } \\
3 \text { hospitals }\end{array}$ & $\begin{array}{l}\text { Hospital } 1=2441 \\
\text { Hospital } 2=26520 \\
\text { Hospital } 3=45785\end{array}$ & $\geq 65$ & Not reported & $\begin{array}{l}H 1=23 \% \\
H 2=20 \% \\
H 3=18 \%\end{array}$ \\
\hline $\begin{array}{l}\text { Baillie et } a l^{18} \\
\text { USA }\end{array}$ & Prediction model & $\begin{array}{l}\text { 30-day all-cause } \\
\text { readmissions }\end{array}$ & $\begin{array}{l}\text { Retrospective and } \\
\text { prospective cohort } \\
3 \text { hospitals }\end{array}$ & $\begin{array}{l}\text { Retrospective: } 120396 \\
\text { discharges } \\
\text { prospective validation }\end{array}$ & Not reported-adult & $\begin{array}{l}\text { August 2009- } \\
\text { September } \\
2012\end{array}$ & $\begin{array}{l}\text { Retrospective: } \\
14.4 \% \text {; } \\
\text { Prospective: } \\
15.1 \%\end{array}$ \\
\hline $\begin{array}{l}\text { Choudhry et al }{ }^{12} \\
\text { USA }\end{array}$ & $\begin{array}{l}\text { ACC Admission and } \\
\text { Discharge model }\end{array}$ & $\begin{array}{l}\text { 30-day all-cause } \\
\text { readmissions }\end{array}$ & $\begin{array}{l}\text { Retrospective cohort } \\
8 \text { hospitals }\end{array}$ & $\begin{array}{l}\text { A total of } 126479 \\
\text { patients } \\
94859 \text { derivation set } \\
31619 \text { internal and } \\
6357 \text { external } \\
\text { validation }\end{array}$ & $\begin{array}{l}\text { Mean }=66.01 \\
\text { (readmission) } \\
57.65 \text { (no } \\
\text { readmission) }\end{array}$ & $\begin{array}{l}1 \text { March 2010- } \\
31 \text { July } 2012\end{array}$ & $7.25 \%$ \\
\hline $\begin{array}{l}\text { Gildersleeve and } \\
\text { Cooper }^{19}\end{array}$ & $\begin{array}{l}\text { Risk of readmission score } \\
\text { (RRS) }\end{array}$ & $\begin{array}{l}\text { 30-day all-cause } \\
\text { readmission }\end{array}$ & $\begin{array}{l}\text { Retrospective cohort } \\
1 \text { community }\end{array}$ & $\begin{array}{l}\text { Derivation: } 8700 \\
\text { patients }\end{array}$ & Mean $=60.6$ & 2010 & $14.1 \%$ \\
\hline USA & & & hospital & $\begin{array}{l}\text { Validation: } 8189 \\
\text { patients }\end{array}$ & Mean=65 & 2011 & $14.8 \%$ \\
\hline $\begin{array}{l}\text { Kruse et a }{ }^{R O} \\
\text { USA }\end{array}$ & Unnamed & $\begin{array}{l}\text { 30-day all-cause } \\
\text { readmission }\end{array}$ & $\begin{array}{l}\text { Retrospective cohort } \\
91 \text { hospitals- } \\
\text { Health Facts } \\
\text { Database }\end{array}$ & $\begin{array}{l}463,351 \text { Index } \\
\text { admissions }\end{array}$ & $\geq 18$ & $\begin{array}{l}1 \text { October } \\
2008-31 \\
\text { August } 2010\end{array}$ & $9.7 \%$ \\
\hline $\begin{array}{l}\text { Richmond }{ }^{21} \\
\text { USA }\end{array}$ & Unnamed & $\begin{array}{l}\text { 30-day all-cause } \\
\text { readmission for } \\
\text { patients } \geq 65 \text { years }\end{array}$ & $\begin{array}{l}\text { Retrospective cohort } \\
\text { state-level database }\end{array}$ & $\begin{array}{l}4717 \text { patients split into } \\
\text { a derivation ( } 80 \%) \text { and } \\
\text { validation sample } \\
(20 \%)\end{array}$ & Mean $=77.27$ & $\begin{array}{l}\text { January 2010- } \\
\text { December } \\
2012\end{array}$ & $14.4 \%$ \\
\hline $\begin{array}{l}\text { Shulan et a }{ }^{2} \\
\text { USA }\end{array}$ & Unnamed & $\begin{array}{l}\text { 30-day all-cause } \\
\text { readmission }\end{array}$ & $\begin{array}{l}\text { Retrospective cohort } \\
\text { centralised database }\end{array}$ & $\begin{array}{l}8718 \text { patients } \\
\text { Derivation }(50 \%) \\
\text { Validation }(50 \%)\end{array}$ & $\begin{array}{l}\text { Mean=67.04 } \\
\text { (UHRs); } 66.43 \text { (no } \\
\text { UHRs) }\end{array}$ & 2011 & $16.2 \%$ \\
\hline $\begin{array}{l}\text { van Walraven et a }{ }^{33} \\
\text { Canada }\end{array}$ & $\begin{array}{l}\text { LACE }+ \text { (extension of a } \\
\text { validated index) }\end{array}$ & $\begin{array}{l}\text { 30-day all-cause } \\
\text { readmission }\end{array}$ & $\begin{array}{l}\text { Retrospective cohort } \\
\text { centralised database }\end{array}$ & $\begin{array}{l}499996 \text { patients/ } \\
858410 \text { index } \\
\text { hospitalisations }\end{array}$ & $>18$ & 2004-2009 & $11.8 \%$ \\
\hline $\begin{array}{l}\text { Cotter et al }{ }^{13} \\
\text { UK }\end{array}$ & LACE index (validation) & $\begin{array}{l}\text { 30-day all-cause } \\
\text { readmission }\end{array}$ & $\begin{array}{l}\text { Retrospective cohort } \\
\text { centralised database }\end{array}$ & 507 patients & Mean $=85$ & 2010 & $17.8 \%$ \\
\hline
\end{tabular}




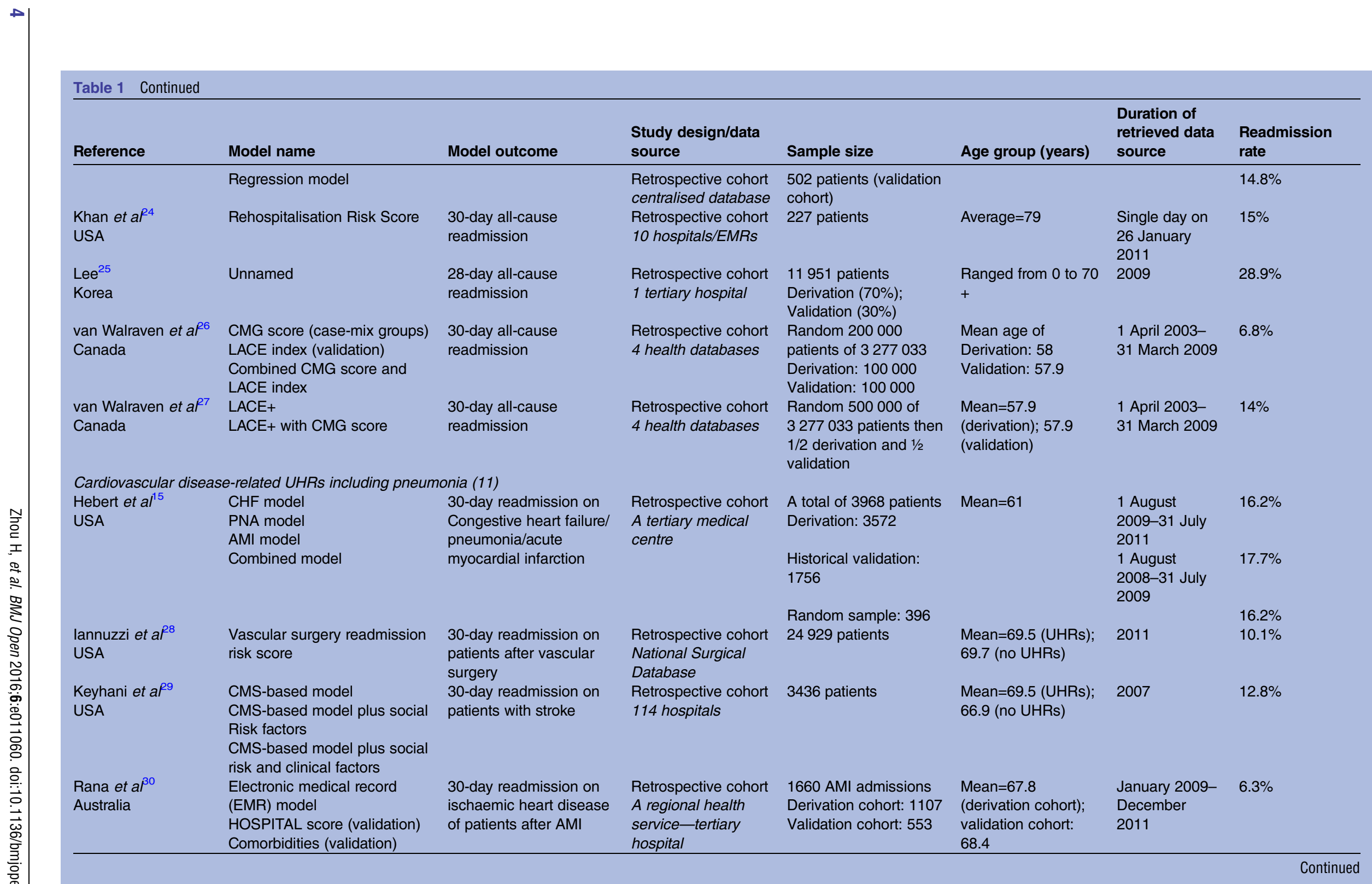

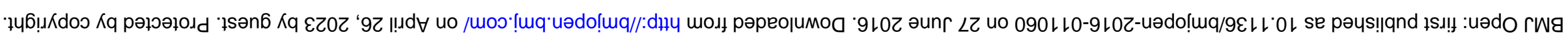




\begin{tabular}{|c|c|c|c|c|c|c|c|}
\hline Reference & Model name & Model outcome & $\begin{array}{l}\text { Study design/data } \\
\text { source }\end{array}$ & Sample size & Age group (years) & $\begin{array}{l}\text { Duration of } \\
\text { retrieved data } \\
\text { source }\end{array}$ & $\begin{array}{l}\text { Readmission } \\
\text { rate }\end{array}$ \\
\hline $\begin{array}{l}\text { Shahian et } a l^{31} \\
\text { USA }\end{array}$ & Unnamed & $\begin{array}{l}\text { 30-day readmission post } \\
\text { coronary artery bypass } \\
\text { grafting }\end{array}$ & $\begin{array}{l}\text { Retrospective cohort } \\
\text { National Database } \\
\text { (846 hospitals) }\end{array}$ & 162572 admissions & $\geq 65$ & 2008-2010 & $12.6-23.6 \%$ \\
\hline \multirow[t]{3}{*}{$\begin{array}{l}\text { Shams et }\left.a\right|^{32} \\
\text { USA }\end{array}$} & $\begin{array}{l}\text { Potentially avoidable } \\
\text { readmission (PAR) }\end{array}$ & $\begin{array}{l}\text { 30-day avoidable } \\
\text { readmission on } \\
\text { pneumonia/HF/AMI/ } \\
\text { COPD }\end{array}$ & $\begin{array}{l}\text { Retrospective cohort } \\
\text { Veterans Health } \\
\text { Administration data } \\
\text { Internal validation }\end{array}$ & 5600 admissions & $\begin{array}{l}\text { HF: mean=71.3 } \\
\text { (PAR); vs } 68.6 \text { (no } \\
\text { UHRs) } \\
\text { AMI: mean=73.3 }\end{array}$ & 2011-2012 & $13.09 \%$ \\
\hline & & & External validation & 478 patients & $\begin{array}{l}\text { (PAR) vs } 69.3 \text { (no } \\
\text { UHRs) }\end{array}$ & $\begin{array}{l}\text { August and } \\
\text { September } \\
2012\end{array}$ & \\
\hline & $\begin{array}{l}\text { CMS endorsed model } \\
\text { (validation) }\end{array}$ & 30-day readmission & & & & & \\
\hline $\begin{array}{l}\text { Sharif et a }{ }^{\beta 3} \\
\text { USA }\end{array}$ & Unnamed & $\begin{array}{l}30 \text {-day readmission on } \\
\text { patients aged } 40- \\
64 \text { years with COPD }\end{array}$ & $\begin{array}{l}\text { Retrospective cohort } \\
\text { A large national } \\
\text { commercial } \\
\text { insurance database }\end{array}$ & 8263 patients & $\begin{array}{l}\text { Mean=57 (UHRs); } \\
\text { no UHRs-age not } \\
\text { reported }\end{array}$ & $\begin{array}{l}\text { January 2009- } \\
\text { November } \\
2011\end{array}$ & $8.9 \%$ \\
\hline $\begin{array}{l}\text { Lucas et a }{ }^{\beta 4} \\
\text { USA }\end{array}$ & $\begin{array}{l}\text { Complex all-variable model; } \\
\text { parsimonious readmission } \\
\text { score }\end{array}$ & $\begin{array}{l}\text { 30-day readmissions on } \\
\text { patients post general, } \\
\text { vascular, and thoracic } \\
\text { surgery }\end{array}$ & $\begin{array}{l}\text { Retrospective cohort } \\
\text { National Surgery } \\
\text { Database }\end{array}$ & $\begin{array}{l}\text { A total of } 230864 \\
\text { patients } \\
\text { Derivation: } 162159 \\
(70 \%) \text { : Validation: } \\
68705(30 \%)\end{array}$ & Median=56 & 2011 & $\begin{array}{l}5-16 \% \text { across } \\
\text { surgical } \\
\text { specialties }\end{array}$ \\
\hline $\begin{array}{l}\text { Wallmann et } a{ }^{\beta 5} \\
\text { Spain }\end{array}$ & Unnamed & $\begin{array}{l}\text { 30-day readmission on } \\
\text { cardiac-related disease }\end{array}$ & $\begin{array}{l}\text { Retrospective cohort } \\
1 \text { tertiary centre }\end{array}$ & $\begin{array}{l}35531 \text { admissions } \\
\text { Derivation cohort: } \\
24881 \\
\text { Validation cohort: } \\
10650\end{array}$ & Mean $=67.9$ & 2003-2009 & $\begin{array}{l}\text { Derivation: } 4.4 \% \text {; } \\
\text { Validation: } 4.7 \%\end{array}$ \\
\hline $\begin{array}{l}\text { Wasfy et } a \beta^{\beta 6} \\
\text { USA }\end{array}$ & $\begin{array}{l}\text { Risk score for } 30-\text { day } \\
\text { readmission after } \mathrm{PCl} \\
\text { (parsimonious) }\end{array}$ & $\begin{array}{l}\text { 30-day readmission after } \\
\text { percutaneous coronary } \\
\text { intervention }\end{array}$ & $\begin{array}{l}\text { Retrospective cohort } \\
\text { centralised database }\end{array}$ & $\begin{array}{l}36060 \text { surviving to } \\
\text { discharge }\end{array}$ & $\begin{array}{l}\text { Mean=68.1 (UHRs); } \\
64.3 \text { (no UHRs) }\end{array}$ & $\begin{array}{l}1 \text { October } \\
2005-30 \\
\text { September } 30 \\
2008\end{array}$ & $10.4 \%$ \\
\hline \multirow[t]{2}{*}{$\begin{array}{l}\text { Krumholz et } a P^{\beta 7} \\
\text { USA }\end{array}$} & Claims model & $\begin{array}{l}\text { 30-day readmission on } \\
\text { acute myocardial } \\
\text { infarction (AMI) }\end{array}$ & $\begin{array}{l}\text { Retrospective cohort } \\
\text { Medicare Claims } \\
\text { Database }\end{array}$ & $\begin{array}{l}\text { Derivation cohort: } \\
100465 \\
\text { Validation cohort: } \\
321088\end{array}$ & Mean $=78.7$ & Half of 2006 & $18.9 \%$ \\
\hline & Medical record model & & & $\begin{array}{l}\text { Derivation cohort: } \\
130944 \\
\text { Validation cohort: } \\
130944\end{array}$ & & $\begin{array}{l}2005 \text { and half } \\
\text { of } 2006\end{array}$ & $19.96 \%$ \\
\hline \multicolumn{8}{|c|}{ Cardiovascular disease-related UHRs including pneumonia—heart failure only (11) } \\
\hline $\begin{array}{l}\text { Betihavas et } a p^{\beta 8} \\
\text { Australia }\end{array}$ & Unnamed & $\begin{array}{l}\text { 28-day readmission on } \\
\text { patients with chronic } \\
\text { heart failure }\end{array}$ & $\begin{array}{l}\text { Retrospective cohort } \\
\text { Multicentre }\end{array}$ & $\begin{array}{l}280 \text { patients } \\
94 \text { (no UHRs); } 37 \\
\text { (28-D UHRs) }\end{array}$ & $\begin{array}{l}\text { Mean=69 (no } \\
\text { UHRs); } 79 \text { (UHRs) }\end{array}$ & Not reported & $13 \%$ \\
\hline
\end{tabular}




\begin{tabular}{|c|c|c|c|c|c|c|c|}
\hline Reference & Model name & Model outcome & $\begin{array}{l}\text { Study design/data } \\
\text { source }\end{array}$ & Sample size & Age group (years) & $\begin{array}{l}\text { Duration of } \\
\text { retrieved data } \\
\text { source }\end{array}$ & $\begin{array}{l}\text { Readmission } \\
\text { rate }\end{array}$ \\
\hline $\begin{array}{l}\text { Di Tano et } a p^{\beta 9} \\
\text { Italy }\end{array}$ & Unnamed & $\begin{array}{l}\text { 30-day readmission on } \\
\text { acute HF }\end{array}$ & $\begin{array}{l}\text { Prospective cohort } \\
\text { National Registry } \\
\text { Database }\end{array}$ & 1520 patients & Mean $=72$ & Not reported & $6.25 \%$ \\
\hline $\begin{array}{l}\text { Huynh et } a f^{40} \\
\text { Australia }\end{array}$ & $\begin{array}{l}\text { The non-clinical model } \\
\text { The clinical model } \\
\text { The combined model }\end{array}$ & $\begin{array}{l}\text { 30-day readmission on } \\
\text { HF }\end{array}$ & $\begin{array}{l}\text { Retrospective cohort } \\
\text { state-wide data } \\
\text { linkage }\end{array}$ & $\begin{array}{l}\text { Non-clinical-1537 } \\
\text { patients } \\
\text { Clinical-977 patients } \\
\text { available }\end{array}$ & Mean $=80$ & 2009-2012 & $25.4 \%$ \\
\hline $\begin{array}{l}\text { Raposeiras-Roubin } \\
\text { et } a /^{11} \\
\text { Spain }\end{array}$ & GRACE risk score & $\begin{array}{l}\text { 30-day readmission on } \\
\text { HF after acute coronary } \\
\text { syndrome }\end{array}$ & $\begin{array}{l}\text { Retrospective cohort } \\
\text { A single centre }\end{array}$ & 4429 patients & $\begin{array}{l}\text { Mean=77 (UHRs); } \\
68 \text { (no UHRs) }\end{array}$ & 2004-2010 & $1.3 \%$ \\
\hline $\begin{array}{l}\text { Sudhakar et } a l^{42} \\
\text { USA }\end{array}$ & Readmission Risk score & $\begin{array}{l}\text { 30-day readmission on } \\
\text { patients with } \mathrm{CHF}\end{array}$ & $\begin{array}{l}\text { Retrospective cohort } \\
\text { A tertiary hospital/ } \\
\text { chart review }\end{array}$ & $\begin{array}{l}1046 \text { admissions from } \\
712 \text { patients }\end{array}$ & Mean $=65.2$ & $\begin{array}{l}\text { September } \\
2011 \text {-August } \\
2013\end{array}$ & $35.28 \%$ \\
\hline $\begin{array}{l}\text { Fleming et } a l^{43} \\
\text { USA }\end{array}$ & Unnamed & $\begin{array}{l}\text { 30-day readmission on } \\
\text { patients with HF }\end{array}$ & $\begin{array}{l}\text { Retrospective cohort } \\
1 \text { tertiary medical } \\
\text { centre }\end{array}$ & $\begin{array}{l}3413 \text { admissions } \\
\text { Derivation: } \\
\text { Validation }=3: 1 \\
(2566: 847)\end{array}$ & $\begin{array}{l}\text { Mean }=74 \text { (derivation } \\
\text { cohort); validation } \\
\text { cohort: } 74.6\end{array}$ & $\begin{array}{l}1 \text { October } \\
2007-30 \\
\text { August } 2011\end{array}$ & $\begin{array}{l}24.2 \% \\
\text { (derivation) }\end{array}$ \\
\hline $\begin{array}{l}\text { Wang et } a f^{44} \\
\text { USA }\end{array}$ & LACE index (validation) & $\begin{array}{l}\text { 30-day readmission on } \\
\text { patients with } \mathrm{CHF}\end{array}$ & $\begin{array}{l}\text { Retrospective cohort } \\
\text { An urban public } \\
\text { hospital }\end{array}$ & 253 patients & $\begin{array}{l}\text { Mean: } 57.67 \text { (no } \\
\text { UHRs); } 56.17 \\
\text { (UHRs) }\end{array}$ & $\begin{array}{l}\text { June } 2012- \\
\text { June } 2013\end{array}$ & $24.5 \%$ \\
\hline $\begin{array}{l}\text { Eapen et a } f^{45} \\
\text { USA }\end{array}$ & Unnamed & $\begin{array}{l}\text { 30-day readmission on } \\
\text { heart failure }\end{array}$ & $\begin{array}{l}\text { Retrospective cohort } \\
\text { Centers for } \\
\text { Medicare database }\end{array}$ & $\begin{array}{l}33349 \text { patient } \\
70 \% \text { in derivation } \\
\text { cohort } \\
30 \% \text { in validation } \\
\text { cohort }\end{array}$ & Median=80 & $\begin{array}{l}1 \text { January } \\
2005-31 \\
\text { December } \\
2009\end{array}$ & $22.8 \%$ \\
\hline $\begin{array}{l}\text { Zai et } a f^{46} \\
\text { USA }\end{array}$ & $\begin{array}{l}\text { The telemonitoring-based } \\
\text { readmission model; the } \\
\text { psychosocial readmission } \\
\text { model (validation) }\end{array}$ & $\begin{array}{l}\text { 30-day readmission on } \\
\text { heart failure }\end{array}$ & $\begin{array}{l}\text { Retrospective cohort } \\
\text { Patients enrolled in } \\
\text { the telemonitoring } \\
\text { program }\end{array}$ & 100 patients & Average age of 66.8 & $\begin{array}{l}\text { July 2008- } \\
\text { November } \\
2011\end{array}$ & $38 \%$ \\
\hline $\begin{array}{l}\text { Au et } a f^{47} \\
\text { Canada }\end{array}$ & $\begin{array}{l}\text { Five administrative } \\
\text { data-based models: Charlson; } \\
\text { CMS Krumholz } \\
\text { Keenan; LACE; LACE+ }\end{array}$ & $\begin{array}{l}\text { 30-day readmission on } \\
\text { HF }\end{array}$ & $\begin{array}{l}\text { Retrospective cohort } \\
4 \text { health databases }\end{array}$ & 59652 patients & Mean=76 & $\begin{array}{l}\text { April } 1999 \text { and } \\
2009\end{array}$ & $19 \%$ \\
\hline $\begin{array}{l}\text { Watson et } a l^{48} \\
\text { USA }\end{array}$ & $\begin{array}{l}\text { The psychosocial readmission } \\
\text { model }\end{array}$ & $\begin{array}{l}\text { 30-day readmission on } \\
\text { HF }\end{array}$ & $\begin{array}{l}\text { Retrospective cohort } \\
1 \text { tertiary hospital }\end{array}$ & 729 & Mean=71.4 & $\begin{array}{l}1 \text { October } \\
2007-30 \\
\text { September } \\
2008\end{array}$ & $\begin{array}{l}13.3 \% \text { (all } \\
\text { female) }\end{array}$ \\
\hline \multicolumn{8}{|c|}{ Cardiovascular disease-related UHRs including pneumonia-pneumonia only (2) } \\
\hline $\begin{array}{l}\text { Mather et } a{ }^{49} \\
\text { USA }\end{array}$ & $\begin{array}{l}\text { Hartford Hospital model } \\
\text { CMS Model (validation) }\end{array}$ & $\begin{array}{l}\text { 30-day readmission on } \\
\text { pneumonia }\end{array}$ & $\begin{array}{l}\text { Retrospective cohort } \\
\text { A tertiary hospital }\end{array}$ & 956 index admissions & $\geq 65$ & $\begin{array}{l}\text { January 2009- } \\
\text { March } 2012\end{array}$ & $15.5 \%$ \\
\hline
\end{tabular}


Table 1 Continued

\begin{tabular}{|c|c|c|c|c|c|c|c|}
\hline Reference & Model name & Model outcome & $\begin{array}{l}\text { Study design/data } \\
\text { source }\end{array}$ & Sample size & Age group (years) & $\begin{array}{l}\text { Duration of } \\
\text { retrieved data } \\
\text { source }\end{array}$ & $\begin{array}{l}\text { Readmission } \\
\text { rate }\end{array}$ \\
\hline \multirow[t]{2}{*}{$\begin{array}{l}\text { Lindenauer et } a^{{ }^{0}} \\
\text { USA }\end{array}$} & Administrative claims model & $\begin{array}{l}\text { 30-day readmission on } \\
\text { pneumonia }\end{array}$ & $\begin{array}{l}\text { Retrospective cohort } \\
\text { Medicare enrolment } \\
\text { database }\end{array}$ & $\begin{array}{l}\text { Derivation cohort: } \\
226545 \\
\text { Validation cohort: } \\
762721\end{array}$ & Mean $=80$ & Half of 2006 & $17.4 \%$ \\
\hline & Medical record model & & & 47429 cases & & $\begin{array}{l}\text { Half of } 2006 \\
\text { and } 2005\end{array}$ & $17.0 \%$ \\
\hline \multicolumn{8}{|c|}{ General medical condition-related UHRs (10) } \\
\hline $\begin{array}{l}\text { Shadmi et } a^{F^{1}} \\
\text { Israel }\end{array}$ & $\begin{array}{l}\text { Preadmission Readmission } \\
\text { Detection Model }\end{array}$ & $\begin{array}{l}\text { 30-day readmission on } \\
\text { medical patients }\end{array}$ & $\begin{array}{l}\text { Retrospective cohort } \\
\text { Clalit Health } \\
\text { Services/EMR }\end{array}$ & $\begin{array}{l}\text { Total: } 33639 \\
\text { admissions } \\
\text { Derivation: } 22406 \\
\text { Validation: } 11233\end{array}$ & $\begin{array}{l}\text { Mean=68.2; } 67.5 \\
\text { (no UHRs); } 72.5 \\
\text { (UHRs) }\end{array}$ & $\begin{array}{l}1 \text { January } \\
2010-31 \text { March } \\
2010\end{array}$ & $16.8 \%$ \\
\hline $\begin{array}{l}\text { Tsui et } a^{p^{2}} \\
\text { Hong Kong }\end{array}$ & Unnamed & $\begin{array}{l}\text { 28-day readmission on } \\
\text { elderly medical patients }\end{array}$ & $\begin{array}{l}\text { Retrospective cohort } \\
41 \text { hospitals/EMS }\end{array}$ & $\begin{array}{l}\text { Total: } 327529 \\
\text { episodes } \\
\text { Derivation: } 165216 \\
\text { Validation: } 162313\end{array}$ & $\geq 65$ & $\begin{array}{l}\text { Derivation: } \\
2005 \\
\text { Validation: } \\
2006\end{array}$ & $\begin{array}{l}7.8 \% \\
7.6 \%\end{array}$ \\
\hline $\begin{array}{l}\text { Donzé et } a^{53} \\
\text { USA }\end{array}$ & Unnamed & $\begin{array}{l}\text { 30-day readmission on } \\
\text { medical patients due to } \\
\text { end-of-life care }\end{array}$ & $\begin{array}{l}\text { Retrospective cohort } \\
1 \text { tertiary medical } \\
\text { centre including } 3 \\
\text { hospitals }\end{array}$ & 10275 admissions & $\begin{array}{l}\text { Mean=61.5 (no } \\
\text { UHRs); } 60.8 \\
\text { (potentially } \\
\text { avoidable } \\
\text { readmissions } \\
\text { (PARs) }\end{array}$ & $\begin{array}{l}1 \text { July } 2009-30 \\
\text { June } 2010\end{array}$ & $\begin{array}{l}\text { Total: } 22.3 \% ; 8 \% \\
\text {-PARs }\end{array}$ \\
\hline \multirow[t]{2}{*}{$\begin{array}{l}\text { He et } a P^{4} \\
\text { USA }\end{array}$} & Unnamed & $\begin{array}{l}\text { 30-day readmission on } \\
\text { medical patients and } \\
\text { chronic pancreatitis }(C P)\end{array}$ & $\begin{array}{l}\text { Retrospective cohort } \\
\text { JHH (tertiary centre) } \\
\text { BMC (community } \\
\text { hospital) }\end{array}$ & $\begin{array}{l}\text { Medical patients: } \\
26091(\mathrm{JHH})+16194 \\
\text { (BMC) }\end{array}$ & $\begin{array}{l}\text { Mean=50.3 (JHH) } \\
51.5(\mathrm{BMC})\end{array}$ & $\begin{array}{l}\text { Medical } \\
\text { patients: } \\
\text { January 2012- } \\
\text { April 2013; }\end{array}$ & $\begin{array}{l}11.5 \%(\mathrm{JHH}) \\
8.7 \%(\mathrm{BMC})\end{array}$ \\
\hline & & & & $\begin{array}{l}\text { Patients with CP: } 3218 \\
(\mathrm{JHH})+706(\mathrm{BMC})\end{array}$ & $\begin{array}{l}\text { Mean age: } 51.4 \\
(\mathrm{JHH}) \\
51.4(\mathrm{BMC})\end{array}$ & $\begin{array}{l}\text { CP discharged } \\
\text { from January } \\
2007-A p r i l \\
2013\end{array}$ & $\begin{array}{l}15.6 \%(\mathrm{JHH}) \\
7.8 \%(\mathrm{BMC})\end{array}$ \\
\hline $\begin{array}{l}\text { Taha et a }{ }^{55} \\
\text { USA }\end{array}$ & $\begin{array}{l}\text { Readmission Risk Score } \\
\text { (RRS) }\end{array}$ & $\begin{array}{l}\text { 30-day readmission on } \\
\text { general internal } \\
\text { medicine services }\end{array}$ & $\begin{array}{l}\text { Retrospective cohort } \\
4 \text { teaching and } 2 \\
\text { non-teaching } \\
\text { general internal } \\
\text { medicine services }\end{array}$ & $\begin{array}{l}858 \text { index } \\
\text { hospitalisations } \\
\text { Derivation cohort: } 613 \\
\text { Validation cohort: } 245\end{array}$ & $\begin{array}{l}\text { Mean }=54 \\
\text { (derivation); } \\
\text { validation cohort: } 54\end{array}$ & $\begin{array}{l}1 \text { April } 2010- \\
30 \text { June } 2010\end{array}$ & $16 \%$ \\
\hline $\begin{array}{l}\text { Donzé et } a l^{14} \\
\text { USA }\end{array}$ & HOSPITAL score & $\begin{array}{l}\text { 30-day readmissions on } \\
\text { general medical patients }\end{array}$ & $\begin{array}{l}\text { Retrospective cohort } \\
\text { Multicentre health } \\
\text { services }\end{array}$ & 10731 discharges & Mean $=61.3$ & $\begin{array}{l}1 \text { July } 2009-30 \\
\text { June } 2010\end{array}$ & $8.5 \%$ \\
\hline $\begin{array}{l}\text { Tan et } a^{56} \\
\text { Singapore }\end{array}$ & LACE index (validation) & $\begin{array}{l}\text { 30-day readmission on } \\
\text { general medical patients }\end{array}$ & $\begin{array}{l}\text { Retrospective } \\
\text { The largest tertiary } \\
\text { general hospital }\end{array}$ & 127550 patients & $\geq 21$ & $\begin{array}{l}1 \text { January } \\
2006-31 \\
\text { December } \\
2010\end{array}$ & $4.87-18.43 \%$ \\
\hline
\end{tabular}




\begin{tabular}{|c|c|c|c|c|c|c|c|}
\hline Reference & Model name & Model outcome & $\begin{array}{l}\text { Study design/data } \\
\text { source }\end{array}$ & Sample size & Age group (years) & $\begin{array}{l}\text { Duration of } \\
\text { retrieved data } \\
\text { source }\end{array}$ & $\begin{array}{l}\text { Readmission } \\
\text { rate }\end{array}$ \\
\hline $\begin{array}{l}\text { Billings et } a l^{11} \\
\text { USA }\end{array}$ & PARR-30 & $\begin{array}{l}30 \text { days readmission on } \\
\text { general medical patients }\end{array}$ & $\begin{array}{l}\text { Retrospective cohort } \\
\text { centralised database }\end{array}$ & 576868 admissions & Adult & $\begin{array}{l}1 \text { April } 2008 \\
\text { and } 31 \text { March } \\
2009\end{array}$ & $12.2 \%$ \\
\hline $\begin{array}{l}\text { Zapatero et } a^{F^{7}} \\
\text { Spain }\end{array}$ & SEMI INDEX & $\begin{array}{l}\text { 30-day readmission on } \\
\text { general medical patients }\end{array}$ & $\begin{array}{l}\text { Retrospective cohort } \\
\text { National Health } \\
\text { Database }\end{array}$ & $\begin{array}{l}\text { Derivation cohort: } \\
999089 \text { patients; } \\
\text { Validation cohort: } \\
510588 \text { patients } \\
\text { (internal) }\end{array}$ & $\begin{array}{l}\text { Median=70 for two } \\
\text { cohorts }\end{array}$ & $\begin{array}{l}\text { January 2006- } \\
\text { December } \\
2007 \\
2008\end{array}$ & $12.5 \%$ \\
\hline $\begin{array}{l}\text { Gruneir et } a^{58} \\
\text { Canada } \\
\text { Medical conditio }\end{array}$ & $\begin{array}{l}\text { LACE index (validation) } \\
\text { Rs-cirrhosis only (2) }\end{array}$ & $\begin{array}{l}\text { 30-day readmission on } \\
\text { general medical patients }\end{array}$ & $\begin{array}{l}\text { Retrospective cohort } \\
6 \text { hospitals }\end{array}$ & 26045 patients & $18-105$ & 2007 & $12.6 \%$ \\
\hline $\begin{array}{l}\text { Singal et } a^{59} \\
\text { USA }\end{array}$ & Unnamed & $\begin{array}{l}\text { 30-day readmissions on } \\
\text { patients with cirrhosis }\end{array}$ & $\begin{array}{l}\text { Retrospective cohort } \\
1 \text { large safety-net } \\
\text { hospital }\end{array}$ & $\begin{array}{l}\text { A total of } 838 \text { patients } \\
\text { with } 1291 \text { admissions } \\
\text { Derivation: } 968 \\
\text { Validation: } 323\end{array}$ & Mean $=52.5$ & $\begin{array}{l}\text { January 2008- } \\
\text { December } \\
2009\end{array}$ & $27 \%$ \\
\hline $\begin{array}{l}\text { Volk et a }{ }^{00} \\
\text { USA } \\
\text { Medical conditio }\end{array}$ & $\begin{array}{l}\text { Cirrhosis readmission } \\
\text { prediction model } \\
\text { Rs-chronic kidney disease only }\end{array}$ & $\begin{array}{l}\text { 30-day readmission on } \\
\text { cirrhosis } \\
\text { (1) }\end{array}$ & $\begin{array}{l}\text { Retrospective cohort } \\
1 \text { tertiary hospital }\end{array}$ & 402 patients & $\geq 18$ & $\begin{array}{l}1 \text { July } 2006-1 \\
\text { July } 2009\end{array}$ & $\begin{array}{l}41 \%, 22 \% \text { of } \\
\text { which are PARs }\end{array}$ \\
\hline $\begin{array}{l}\text { Perkins et } a f^{11} \\
\text { USA }\end{array}$ & Unnamed & $\begin{array}{l}\text { 30-day readmission on } \\
\text { patients with CKD } \\
\text { second to HF }\end{array}$ & $\begin{array}{l}\text { Retrospective cohort } \\
2 \text { inpatient facilities }\end{array}$ & $\begin{array}{l}607 \text { patients with } \\
\text { chronic kidney disease }\end{array}$ & $\begin{array}{l}\text { Mean=72.3 (UHRs); } \\
74.1 \text { (no UHRs) }\end{array}$ & $\begin{array}{l}1 \text { July 2004-28 } \\
\text { February } 2010\end{array}$ & $19.1 \%$ \\
\hline \multicolumn{8}{|c|}{ Medical condition UHRs-HIV only (1) } \\
\hline $\begin{array}{l}\text { Nijhawan et a }{ }^{{ }^{2}} \\
\text { USA }\end{array}$ & Unnamed & $\begin{array}{l}\text { 30-day readmission on } \\
\text { HIV-infected patients }\end{array}$ & $\begin{array}{l}\text { Retrospective cohort } \\
1 \text { tertiary hospital }\end{array}$ & $\begin{array}{l}2402 \text { index admissions } \\
\text { randomly split }(1 / 2) \\
\text { into derivation vs } \\
\text { validation }\end{array}$ & Mean $=43$ & $\begin{array}{l}\text { March 2006- } \\
\text { November } \\
2008\end{array}$ & $24.4 \%$ \\
\hline \multicolumn{8}{|c|}{ Medical condition UHRs-acute pancreatitis (1) } \\
\hline $\begin{array}{l}\text { Whitlock et } a^{{ }^{3}} \\
\text { USA }\end{array}$ & Unnamed & $\begin{array}{l}\text { 30-day readmission on } \\
\text { acute pancreatitis }\end{array}$ & $\begin{array}{l}\text { Retrospective cohort } \\
2 \text { hospitals }\end{array}$ & $\begin{array}{l}\text { Derivation cohort: } 248 \\
\text { Validation cohort: } 198\end{array}$ & $\begin{array}{l}\text { Mean }=51.6 \\
\text { derivation } \\
\text { Validation: } 52.3\end{array}$ & $\begin{array}{l}1 \text { June 2005- } \\
31 \text { December } \\
2007 \\
1 \text { January } \\
2008-31 \\
\text { October } 2009\end{array}$ & $\begin{array}{l}19 \% \\
23 \%\end{array}$ \\
\hline \multicolumn{8}{|c|}{ Surgical condition-related UHRs (6) } \\
\hline $\begin{array}{l}\text { Taber et a }{ }^{4} \\
\text { USA }\end{array}$ & $\begin{array}{l}\text { 30DRA with fixed variable vs } \\
\text { 30DRA with fixed variables } \\
\text { and dynamic clinical data }\end{array}$ & $\begin{array}{l}\text { 30-day readmission on } \\
\text { patients following kidney } \\
\text { transplantation }\end{array}$ & $\begin{array}{l}\text { Retrospective cohort } \\
\text { An institution }\end{array}$ & $\begin{array}{l}1147 \text { patients } \\
\text { Derivation; internal } \\
\text { validation using } \\
\text { random iteration of } \\
50 \% \text { sampling }\end{array}$ & $\begin{array}{l}\text { Mean=51 (no } \\
\text { UHRs); } 52 \text { (UHRs) }\end{array}$ & 2005-2012 & $11 \%$ \\
\hline $\begin{array}{l}\text { Lawson et a }{ }^{65} \\
\text { USA }\end{array}$ & $\begin{array}{l}\text { Unnamed } \\
\text { (demographic, preoperative } \\
\text { and postoperative risk factors) }\end{array}$ & $\begin{array}{l}\text { 30-day readmission on } \\
\text { patients following } \\
\text { colectomy }\end{array}$ & $\begin{array}{l}\text { Retrospective cohort } \\
\text { NSQIP }\end{array}$ & 12981 patients & $\geq 65$ & 2005-2008 & $13.5 \%$ \\
\hline
\end{tabular}




\begin{tabular}{|c|c|c|c|c|c|c|c|}
\hline Reference & Model name & Model outcome & $\begin{array}{l}\text { Study design/data } \\
\text { source }\end{array}$ & Sample size & Age group (years) & $\begin{array}{l}\text { Duration of } \\
\text { retrieved data } \\
\text { source }\end{array}$ & $\begin{array}{l}\text { Readmission } \\
\text { rate }\end{array}$ \\
\hline $\begin{array}{l}\text { lannuzzi et } a^{66} \\
\text { USA }\end{array}$ & $\begin{array}{l}\text { Endocrine surgery } \\
\text { Readmission Risk Score }\end{array}$ & $\begin{array}{l}\text { 30-day readmission on } \\
\text { patients following } \\
\text { cervical endocrine } \\
\text { operations }\end{array}$ & $\begin{array}{l}\text { Retrospective cohort } \\
\text { NSQIP_a large } \\
\text { national clinical } \\
\text { database }\end{array}$ & $\begin{array}{l}34046 \text { cases } \\
\text { Derivation and } \\
\text { validation cohort } \\
\text { (numbers were not } \\
\text { specified) }\end{array}$ & $\begin{array}{l}\text { Mean=54 (no } \\
\text { UHRs); } 55 \text { (UHRs) }\end{array}$ & $2011-2012$ & $2.8 \%$ \\
\hline $\begin{array}{l}\text { Mesko et } a^{77} \\
\text { USA }\end{array}$ & Unnamed & $\begin{array}{l}\text { 30-day readmission on } \\
\text { total hip and knee } \\
\text { arthroplasty }\end{array}$ & $\begin{array}{l}\text { Retrospective cohort } \\
\text { A readmission } \\
\text { database }\end{array}$ & $\begin{array}{l}1291 \text { admissions/1236 } \\
\text { patients }\end{array}$ & $\begin{array}{l}\text { Mean=65.6 (UHRs); } \\
68.3 \text { (no UHRs) }\end{array}$ & $\begin{array}{l}1 \text { May } 2010-30 \\
\text { April } 2011\end{array}$ & $3.6 \%$ \\
\hline $\begin{array}{l}\text { Moore et } a^{{ }^{8}} \\
\text { Canada }\end{array}$ & $\begin{array}{l}\text { Unnamed (quality indicator } \\
\text { based) }\end{array}$ & $\begin{array}{l}\text { 30-day readmission on } \\
\text { trauma }\end{array}$ & $\begin{array}{l}\text { Retrospective cohort } \\
57 \text { trauma centres }\end{array}$ & 57524 patients & $\geq 16$ & $\begin{array}{l}1 \text { April 2005- } \\
28 \text { February } \\
2010\end{array}$ & $6.6 \%$ \\
\hline $\begin{array}{l}\text { Graboyes et a }{ }^{69} \\
\text { USA }\end{array}$ & Unnamed & $\begin{array}{l}\text { 30-day readmission on } \\
\text { otolaryngology patients }\end{array}$ & $\begin{array}{l}\text { Retrospective cohort } \\
\text { A tertiary hospital }\end{array}$ & $\begin{array}{l}1058 \text { patients-1271 } \\
\text { hospital admissions }\end{array}$ & $\begin{array}{l}\text { Mean=52 (no } \\
\text { UHRs); } 56 \text { (UHRs) }\end{array}$ & $\begin{array}{l}1 \text { January } \\
2011-31 \\
\text { December } \\
2011\end{array}$ & $7.3 \%$ \\
\hline \multicolumn{8}{|c|}{ Mental health condition-related UHRs (1) } \\
\hline $\begin{array}{l}\text { Vigod et al } \\
\text { Canada }\end{array}$ & READMIT (41 points) & $\begin{array}{l}\text { 30-day readmission after } \\
\text { discharge from acute } \\
\text { psychiatric units }\end{array}$ & $\begin{array}{l}\text { Retrospective cohort } \\
\text { National health data }\end{array}$ & $\begin{array}{l}\text { Derivation: } 32749 \\
\text { patients } \\
\text { Validation: } 32750 \\
\text { patients }\end{array}$ & $\begin{array}{l}\text { Median=41 (UHRs); } \\
44 \text { (no UHRs) }\end{array}$ & $\begin{array}{l}1 \text { April 2008- } \\
31 \text { March } 2011\end{array}$ & $8.42-10 \%$ \\
\hline
\end{tabular}

ACS, acute coronary syndrome; AMI, acute myocardial infarction; AP, acute pancreatitis, CHF, congestive heart failure; CKD, chronic kidney disease; COPD, common obstructive pulmonary disease; EMRs, electronic medical records; GRACE, global registry of acute coronary events; HF, heart failure; PCI, percutaneous coronary intervention; PREADM, preadmission readmission detection model; PNA, peptide nucleic acid. 
Table 2 Performance of predictive models for 28-day or 30-day unplanned hospital readmissions (UHRs)

\begin{tabular}{|c|c|c|c|c|c|c|c|c|}
\hline Reference & Model name & Discrimination (ROC) & $\begin{array}{l}\text { Calibration } \\
\text { (H\&L) }\end{array}$ & $\begin{array}{l}\text { Threshold } \\
(\%)\end{array}$ & $\begin{array}{l}\text { Sensitivity } \\
(\%)\end{array}$ & $\begin{array}{l}\text { Specificity } \\
(\%)\end{array}$ & $\begin{array}{l}\text { PPV } \\
(\%)\end{array}$ & $\begin{array}{l}\text { NPV } \\
(\%)\end{array}$ \\
\hline \multicolumn{9}{|l|}{ All-cause UHRs (14) } \\
\hline \multirow{3}{*}{ Escobar et al ${ }^{16}$} & ED 30 & Validation: 0.739 & 0.40 & $\geq 20$ & & & & \\
\hline & Discharge 30 & Validation: 0.756 & 0.60 & $\geq 30$ & & & & \\
\hline & LACE (validation) & Validation: 0.729 & 0.40 & $\geq 60$ & & & & \\
\hline \multirow[t]{4}{*}{ Yu et al ${ }^{17}$} & Institution-specific prediction & 0.74 (hospital 2) & & & & & & \\
\hline & model & 0.64 (at admission) & & & & & & \\
\hline & & 0.72 (after discharge) & & & & & & \\
\hline & LACE (validation) & 0.55 (hospital 2) & & & & & & \\
\hline \multirow[t]{2}{*}{ Baillie et $a l^{18}$} & Prediction model & Retrospective: 0.62 & & & 40 & 85 & 31 & 89 \\
\hline & & Prospective: 0.61 & & & 39 & 84 & 30 & 89 \\
\hline \multirow[t]{10}{*}{ Choudhry et al ${ }^{12}$} & ACC Admission Model & Derivation data set: 0.76 & Derivation data set: & 11 & 70 & 71 & & \\
\hline & & Internal validation: 0.75 & $36.0(p<0.001)$ & & & & & \\
\hline & & $\begin{array}{l}\text { Average (500 simulations in } \\
\text { derivation data set): } 0.76\end{array}$ & $\begin{array}{l}\text { Internal validation } \\
\text { data set: } 23.5\end{array}$ & & & & & \\
\hline & & External validation data set with & $(p=0.0027)$ & & & & & \\
\hline & & recalibration: 0.76 & $\begin{array}{l}\text { External validation } \\
\text { with recalibration: } 6.1 \\
(p=0.641 \text { ) }\end{array}$ & & & & & \\
\hline & ACC Discharge Model & Derivation data set: 0.78 & Derivation: 31.1 & 11 & 70 & 71 & & \\
\hline & & Internal validation: 0.77 & $(p<0.001)$ & & & & & \\
\hline & & Average: 0.78 & Internal validation: & & & & & \\
\hline & & External validation data set with & $19.9(p=0.01)$ & & & & & \\
\hline & & recalibration: 0.78 & $\begin{array}{l}\text { External validation } \\
\text { with recalibration: } \\
14.3(p=0.074)\end{array}$ & & & & & \\
\hline \multirow{2}{*}{$\begin{array}{l}\text { Gildersleeve and } \\
\text { Cooper }^{19}\end{array}$} & Risk of readmission score & Derivation cohort: 0.74 & $21.6(p=0.006)$ & 14 & 74.9 & 54.4 & 22.2 & 92.6 \\
\hline & (RRS) & Validation cohort: 0.70 & & & 79.2 & 55.4 & 22.6 & 94.2 \\
\hline \multirow[t]{2}{*}{ Kruse et $a R^{0}$} & Unnamed & Derivation set: 0.668 & & & & & & \\
\hline & & Validation set: 0.657 & & & & & & \\
\hline Richmond $^{21}$ & Unnamed & 0.60 & & & 47 & 78 & & \\
\hline \multirow[t]{2}{*}{ Shulan et a ${ }^{22}$} & Unnamed & Derivation cohort: 0.80 & & & & & & \\
\hline & & Validation cohort: 0.70 & & & & & & \\
\hline \multirow[t]{2}{*}{ van Walraven et $a^{23}$} & LACE + (extension of a & 0.768 ( 1 hospitalisation per patient) & $\mathrm{H}-\mathrm{L} \chi^{2} 50.3$ & & & & & \\
\hline & validated index) & 0.730 (all hospitalisations) & $H-L \chi^{2} 10972$ & & & & & \\
\hline \multirow{2}{*}{ Cotter et al $1^{13}$} & LACE index (validation) & 0.55 & & & & & & \\
\hline & Regression model & 0.57 & & 47 & 54 & 47 & & \\
\hline
\end{tabular}




\begin{tabular}{|c|c|c|c|c|c|c|c|c|}
\hline Reference & Model name & Discrimination (ROC) & $\begin{array}{l}\text { Calibration } \\
(\mathrm{H} \& L)\end{array}$ & $\begin{array}{l}\text { Threshold } \\
(\%)\end{array}$ & $\begin{array}{l}\text { Sensitivity } \\
(\%)\end{array}$ & $\begin{array}{l}\text { Specificity } \\
(\%)\end{array}$ & $\begin{array}{l}\text { PPV } \\
(\%)\end{array}$ & $\begin{array}{l}\text { NPV } \\
(\%)\end{array}$ \\
\hline \multicolumn{9}{|l|}{ All-cause UHRs (14) } \\
\hline \multirow[t]{3}{*}{ Khan et $a P^{4}$} & Rehospitalisation risk score & & & 19 & 97 & 28 & 19 & 98 \\
\hline & & & & 21 & 58 & 63 & 21 & 90 \\
\hline & & & & 27 & 42 & 81 & 27 & 89 \\
\hline $\mathrm{Lee}^{25}$ & Unnamed & $\begin{array}{l}\text { ROC was graphically illustrated, but } \\
\text { no actual number was reported }\end{array}$ & & & & & & \\
\hline \multirow[t]{3}{*}{ van Walraven et $a^{R 6}$} & CMG Score & 0.637 & $\mathrm{p}=0.0079$ & & & & & \\
\hline & LACE index (validation) & 0.72 & $P<0.0001$ & & & & & \\
\hline & $\begin{array}{l}\text { Combined CMG Score and } \\
\text { LACE }\end{array}$ & 0.743 & $p<0.0001$ & & & & & \\
\hline \multirow[t]{2}{*}{ van Walraven et $a^{77}$} & LACE+ (validation) & 0.743 & & & & & & \\
\hline & LACE+ with CMG score & 0.753 & & & & & & \\
\hline \multicolumn{9}{|c|}{ Cardiovascular disease-related UHRs including pneumonia (11) } \\
\hline \multirow[t]{4}{*}{ Hebert et a $\left.\right|^{15}$} & CHF model & Derivation cohort: $0.64-0.73$ & $p>0.05$ & & & & & \\
\hline & PNA model & Historical validation: $0.61-0.68$ & & & & & & \\
\hline & AMI model & Random sample combined: & & & & & & \\
\hline & Combined model & $0.63-0.76$ & & & & & & \\
\hline \multirow[t]{2}{*}{ lannuzzi et $a{ }^{28}$} & Vascular surgery readmission & Derivation dataset: 0.67 & 0.09 & & & & & \\
\hline & risk score & Validation dataset: 0.64 & 0.66 & & & & & \\
\hline \multirow[t]{3}{*}{ Keyhani et $a$ F $^{29}$} & CMS-based model & 0.636 & 0.866 & & & & & \\
\hline & $\begin{array}{l}\text { CMS-based model plus } \\
\text { social risk factors }\end{array}$ & 0.646 & 0.462 & & & & & \\
\hline & $\begin{array}{l}\text { CMS-based model plus } \\
\text { social risk and clinical factors }\end{array}$ & 0.661 & 0.856 & & & & & \\
\hline \multirow[t]{3}{*}{ Rana et $a{ }^{\beta 0}$} & EMR model & 0.78 & & 5 & 65 & 78 & 21 & 83.6 \\
\hline & HOSPITAL score (validation) & 0.60 & & & 62 & 50 & 13 & 78.9 \\
\hline & Comorbidities (validation) & 0.53 & & & & 65 & 45 & \\
\hline Shahian et $a{ }^{\beta 1}$ & Unnamed & 0.648 & & & & & & \\
\hline \multirow[t]{2}{*}{ Shams et $a^{\beta 2}$} & $\begin{array}{l}\text { Potentially avoidable } \\
\text { readmission (PAR) }\end{array}$ & $\begin{array}{l}\text { Retrospective cohort: } 0.836 \\
\text { Validation internal: } 0.818 / \text { external: } \\
0.809\end{array}$ & & & 91.95 & 97.65 & 86.61 & 98.65 \\
\hline & $\begin{array}{l}\text { CMS endorsed model } \\
\text { (validation) }\end{array}$ & 0.63 & & & & & & \\
\hline
\end{tabular}


Table 2 Continued

\begin{tabular}{|c|c|c|c|c|c|c|c|c|}
\hline Reference & Model name & Discrimination (ROC) & $\begin{array}{l}\text { Calibration } \\
(\mathrm{H} \& L)\end{array}$ & $\begin{array}{l}\text { Threshold } \\
(\%)\end{array}$ & $\begin{array}{l}\text { Sensitivity } \\
(\%)\end{array}$ & $\begin{array}{l}\text { Specificity } \\
(\%)\end{array}$ & $\begin{array}{l}\text { PPV } \\
(\%)\end{array}$ & $\begin{array}{l}\text { NPV } \\
(\%)\end{array}$ \\
\hline \multicolumn{9}{|c|}{ Cardiovascular disease-related UHRs including pneumonia (11) } \\
\hline Sharif et $a \beta^{\beta 3}$ & $\begin{array}{l}\text { Unnamed (basic model vs } \\
\text { final model) }\end{array}$ & $\begin{array}{l}\text { Basic model (patient characteristics } \\
\text { only): } 0.677 ; \text { final model (additional } \\
\text { provider-level and system-level } \\
\text { factors) } \\
\text { Derivation set: } 0.717 \\
\text { Validation set: } 0.73\end{array}$ & & & & & & \\
\hline Wallmann et $a{ }^{\beta 5}$ & Unnamed & 0.75 & & 4 & 66 & 70 & 10 & 98 \\
\hline Wasfy et $a^{\beta 6}$ & $\begin{array}{l}\text { Risk score for 30-day } \\
\text { readmission after PCl } \\
\text { (parsimonious) }\end{array}$ & Validation data set: 0.67 & & $>24$ & & & & \\
\hline \multirow[t]{11}{*}{ Lucas et a ${ }^{\beta 4}$} & Complex all-variable model & $\begin{array}{l}\text { Derivation data set: } 0.721 \\
\text { Validation data set: } 0.724\end{array}$ & & & & & & \\
\hline & $\begin{array}{l}\text { Parsimonious readmission } \\
\text { score }\end{array}$ & $\begin{array}{l}\text { Derivation data set: } 0.696 \\
\text { Validation data set: } 0.702\end{array}$ & & 1.2 & 100 & 0 & 8 & / \\
\hline & & & & 2.4 & 99 & 6 & 8 & 99 \\
\hline & & & & 4.7 & 92 & 28 & 10 & 98 \\
\hline & & & & 8 & 77 & 52 & 12 & 97 \\
\hline & & & & 11.8 & 55 & 73 & 15 & 95 \\
\hline & & & & 14.6 & 37 & 85 & 17 & 94 \\
\hline & & & & 17.2 & 21 & 92 & 19 & 93 \\
\hline & & & & 20.3 & 9 & 97 & 21 & 93 \\
\hline & & & & 22.2 & 2 & 100 & 22 & 92 \\
\hline & & & & 40 & 0 & 100 & 40 & 92 \\
\hline \multirow[t]{3}{*}{ Krumholz et a $\beta^{7}$} & Claims model & $\begin{array}{l}\text { Derivation cohort: } 0.63 \\
\text { Validation cohort: } 0.62-0.63\end{array}$ & & & & & & \\
\hline & Medical record model & Derivation cohort: 0.58 & & & & & & \\
\hline & & Validation cohort: 0.59 & & & & & & \\
\hline \multicolumn{9}{|c|}{ Cardiovascular disease-related UHRs including pneumonia - heart failure only (11) } \\
\hline Betihavas et $a^{\beta 8}$ & Unnamed & 0.8 & & & & & & \\
\hline Di Tano et $a p^{\beta 9}$ & Unnamed & 0.695 & & & & & & \\
\hline \multirow[t]{3}{*}{ Huynh et $a f^{00}$} & The non-clinical model & 0.66 & & & & & & \\
\hline & The clinical model & 0.72 & & & & & & \\
\hline & The combined model & 0.76 & & & & & & \\
\hline $\begin{array}{l}\text { Raposeiras-Roubin } \\
\text { et } \mathrm{a}^{41}\end{array}$ & The GRACE risk score & 0.79 & $\mathrm{p}=0.83$ & 37.9 & 82.5 & 62.8 & 5.6 & 99.1 \\
\hline
\end{tabular}


Table 2 Continued

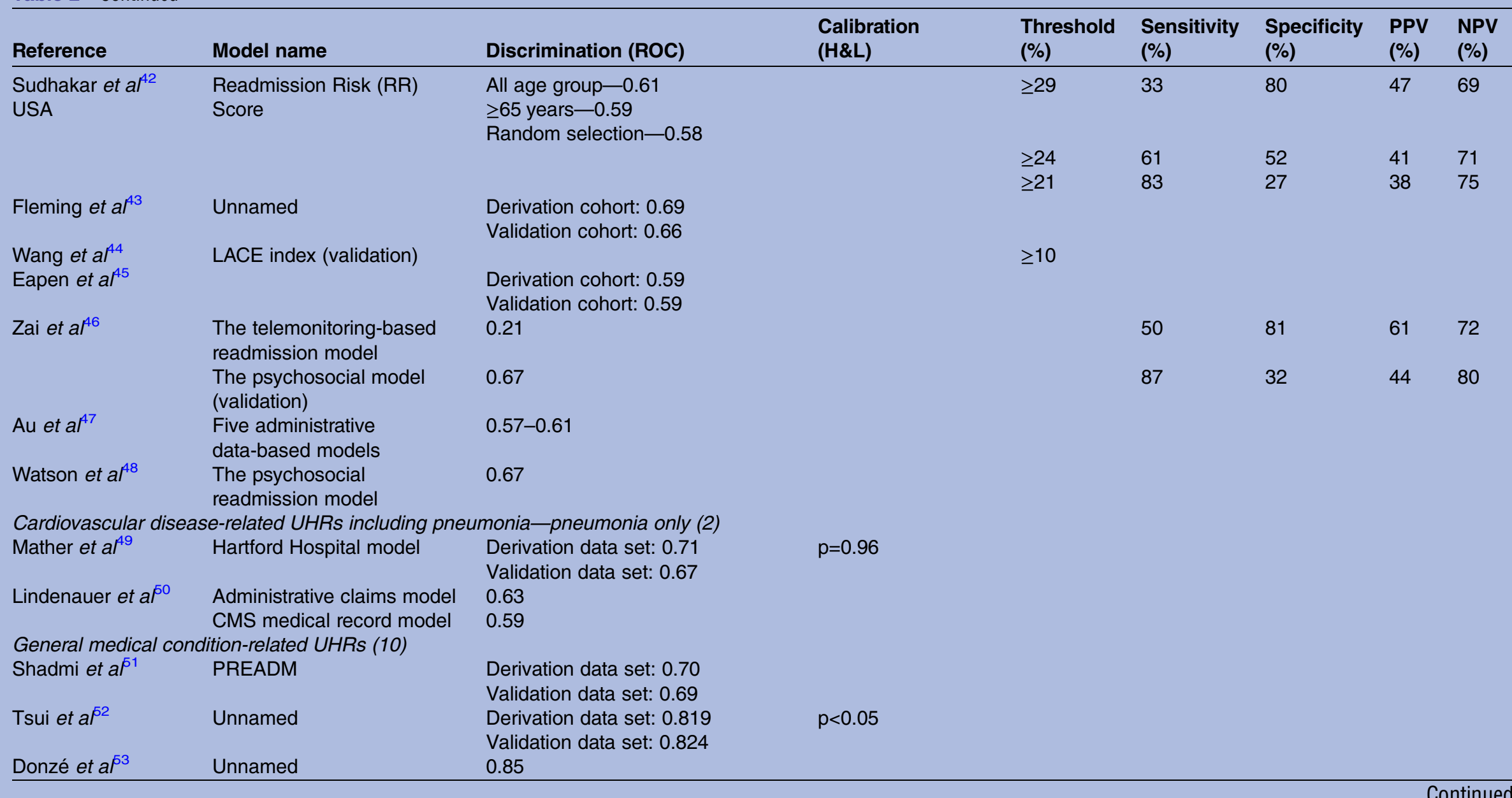




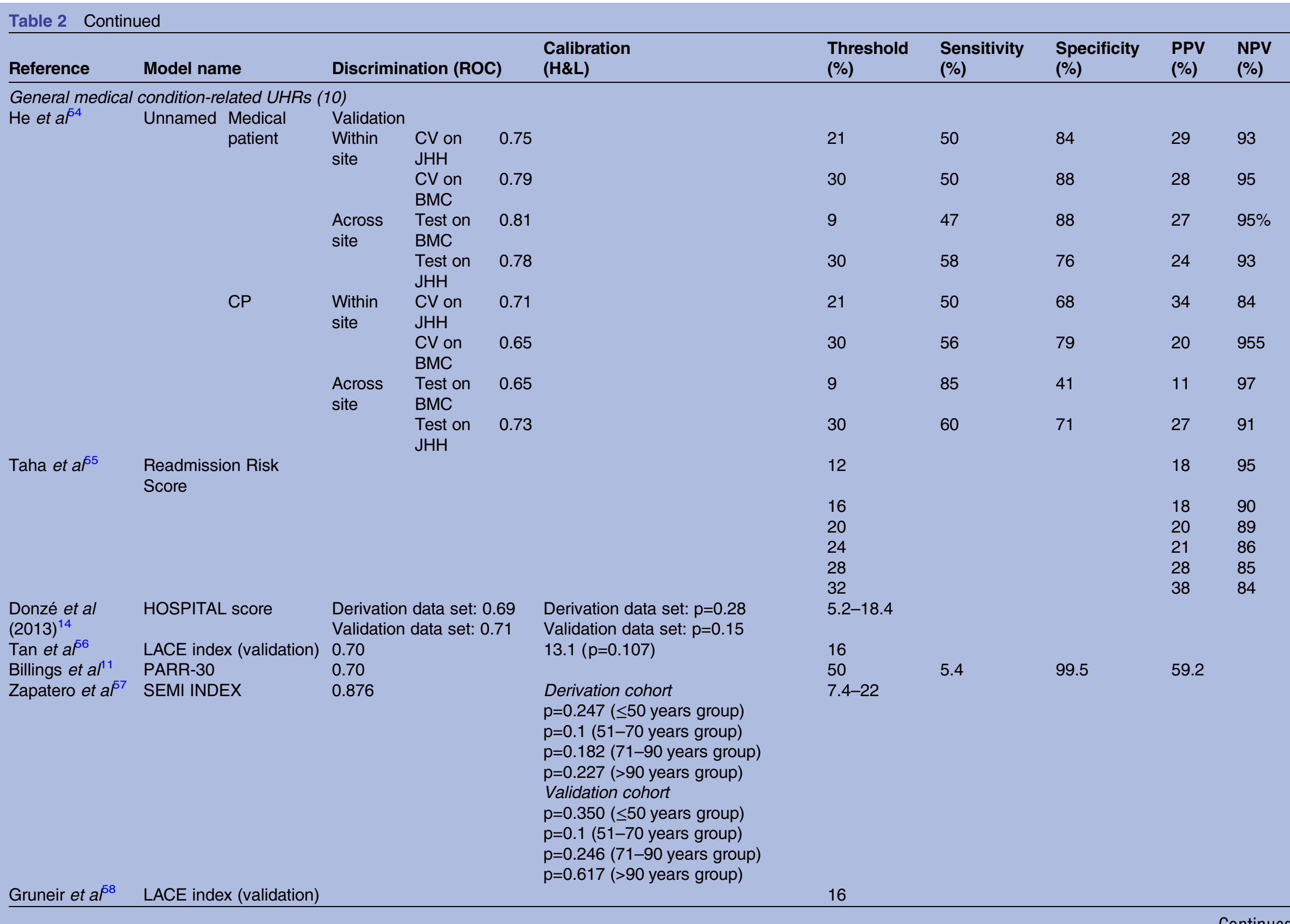




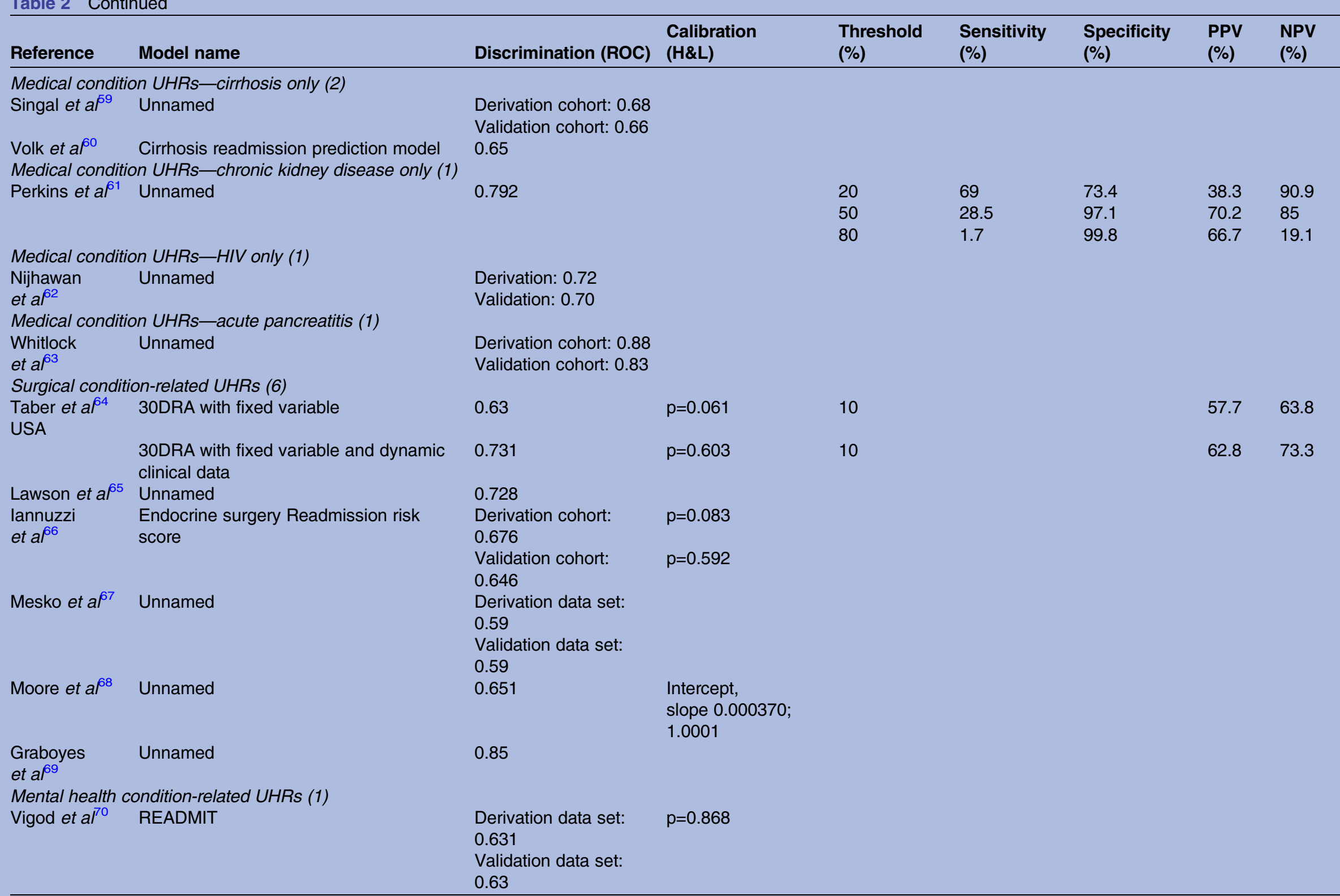

$\overline{\mathrm{NPV}}$, negative predictive value; PPV, positive predictive value; ROC, receiver operating characteristic. 
Model name

\begin{tabular}{|c|c|c|}
\hline \multirow{2}{*}{\multicolumn{3}{|c|}{ All-cause UHRs (14) }} \\
\hline & & \\
\hline \multirow[t]{2}{*}{ Escobar et al ${ }^{16}$} & ED 30 and Discharge 30 & \\
\hline & LACE index (validation) & \\
\hline Yu et $a 1^{17}$ & Institution-specific prediction model & \\
\hline Baillie et $a f^{18}$ & Prediction model & \\
\hline Choudhry et al' ${ }^{2}$ & ACC Admission and Discharge Model & \\
\hline Gildersleeve and Cooper ${ }^{19}$ & Risk of Readmission Score (RRS) & \\
\hline Kruse et a ${ }^{20}$ & Unnamed & \\
\hline Richmond $^{21}$ & Unnamed & \\
\hline Shulan et a $P^{2}$ & Unnamed & \\
\hline van Walraven et $a P^{3}$ & LACE+ (validation) & \\
\hline \multirow[t]{2}{*}{ Cotter et $a l^{13}$} & LACE index (validation) & \\
\hline & Regression model & \\
\hline Khan et $a f^{24}$ & Rehospitalisation Risk Score & \\
\hline $\mathrm{Lee}^{25}$ & Unnamed & \\
\hline \multicolumn{3}{|l|}{ All-cause UHRs (14) } \\
\hline \multirow[t]{3}{*}{ van Walraven et a ${ }^{R^{6}}$} & CMG score & \\
\hline & LACE (validation) & \\
\hline & Combined CMG and LACE & \\
\hline \multirow[t]{2}{*}{ van Walraven et $a^{P^{7}}$} & LACE+ (validation) & \\
\hline & Combined CMG and LACE+ & \\
\hline \multicolumn{3}{|c|}{ Cardiovascular disease-related UHRs including pneumonia (11) } \\
\hline \multirow[t]{4}{*}{ Hebert et al $1^{15}$} & CHF model & \\
\hline & PNA model & \\
\hline & AMI model & \\
\hline & Combined model & \\
\hline lannuzzi et ap ${ }^{8}$ & Vascular surgery readmission risk score & \\
\hline \multirow{3}{*}{ Keyhani et a $P^{9}$} & CMS-based Model & \\
\hline & CMS-based Model plus social risk factors & \\
\hline & CMS-based model plus social risk and clinical factors & \\
\hline Rana et $a^{\beta 0}$ & EMR Model & \\
\hline Shahian et $a \beta^{\beta 1}$ & Unnamed & \\
\hline \multirow[t]{2}{*}{ Shams et $a^{\beta 2}$} & 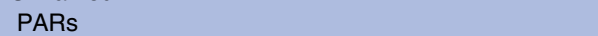 & \\
\hline & CMS endorsed model (validation) & \\
\hline Sharif et $a l^{33}$ & Unnamed & \\
\hline \multirow[t]{2}{*}{ Lucas et $a \beta^{34}$} & Complex all-variable model & \\
\hline & Parsimonious readmission score & \\
\hline Wallmann et a ${ }^{35}$ & & \\
\hline \multirow{2}{*}{ Krumholz et $a \beta^{\beta 7}$} & $\begin{array}{l}\text { Risk score after PCI (parsimonious) } \\
\text { Claims model (administrative) }\end{array}$ & \\
\hline & $\begin{array}{l}\text { Claims model (administrative) } \\
\text { Medical record model }\end{array}$ & \\
\hline
\end{tabular}

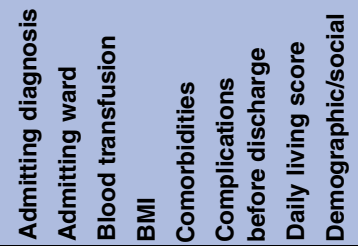

\section{$\checkmark d$}

(vific prediction model
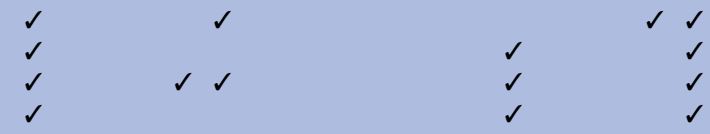

ACC Admission and Discharge Mode

Choudhry et $a^{12}{ }^{12}$ Gooper $^{19}$ Risk of Readmission Score (RRS)

Kruse et a PO Unnamed $^{20} \quad$ Unn

Shulan et a P $^{2}$

van Walraven et $a^{P^{3}} \quad \mathrm{LACE}+$ (validation)

Cotter et $a^{13}{ }^{13}$ LACE index (validation)

Regression model

Rehospitalisation Risk Score

Unnamed

ined CMG and LACE-

Cardiovascular disease-related UHRs including pneumonia (11)

AMI model

Vascular surgery readmission risk score

MS-based Model

S-based model plus social risk and clinical factors $\checkmark$

Parsimonious readmission score

Unnamed

(administrative) 
Reference

Model name

Cardiovascular disease-related UHRs including pneumonia — heart failure only (11)

Betihavas et al ${ }^{\beta 8} \quad$ Unnamed

Di Tano et a $\beta^{\beta 9} \quad$ Unnamed

Huynh et $a f^{40} \quad$ Non-clinical model

Clinical model

Combined model

Raposeiras-Roubin et $a f^{41}$ The GRACE Risk Score

Sudhakar et $\mathrm{a}^{{ }^{2}} \quad$ Readmission Risk Score

USA

Fleming et $a f^{43} \quad$ Unnamed

Wang et $a^{f^{4}} \quad$ LACE index (validation)

Eapen et $a^{45} \quad$ Unnamed

Zai et $a^{\mu^{6}} \quad$ The telemonitoring based readmission model

The psychosocial readmission model (validation)

Au et $a f^{47} \quad$ Charlson (validation)

CMS Krumholz (validation)

Keenan (validation)

LACE (validation)

$\mathrm{LACE}+$ (validation)

Watson et $\mathrm{al}^{48} \quad$ The psychosocial readmission model

Cardiovascular disease-related UHRs including pneumonia-pneumonia only (2)

Mather et $a f^{49} \quad$ Hartford Hospital Model

$\begin{array}{ll} & \text { CMS Model (validation) } \\ \text { Lindenauer et } a^{50} & \text { Claims model (administrative) }\end{array}$

General medical condition UHRs (10)

Shadmi et a F $^{1} \quad$ PREADM

Tsui et $a^{\Gamma^{2}} \quad$ Unnamed

Donzé et al (2014) ${ }^{53} \quad$ Unnamed

He et $a P^{4} \quad$ Unnamed

Taha et $a^{p^{5}} \quad$ Readmission Risk Score (RRS)

Donzé et al (2013) ${ }^{14} \quad$ HOSPITAL score

Tan et $a^{p^{6}}$ LACE index (validation)

Billings et $a l^{11} \quad$ PARR-30

Zapatero et $a^{F^{7}} \quad$ SEMI INDEX

Gruneir et $a^{p^{8}} \quad$ LACE index (validation)

Medical condition UHRs-cirrhosis only (2)

Singal et a ${ }^{59}$

Volk et a ${ }^{\beta 0} \quad$ Cirrhosis readmission prediction model

Medical condition UHRs-chronic kidney disease (1)

Perkins et a ${ }^{11}$ Unnamed

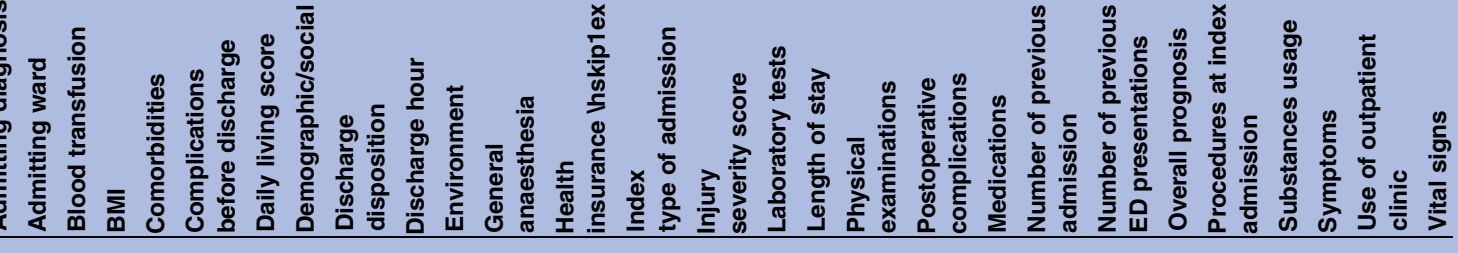

\section{$\checkmark$}




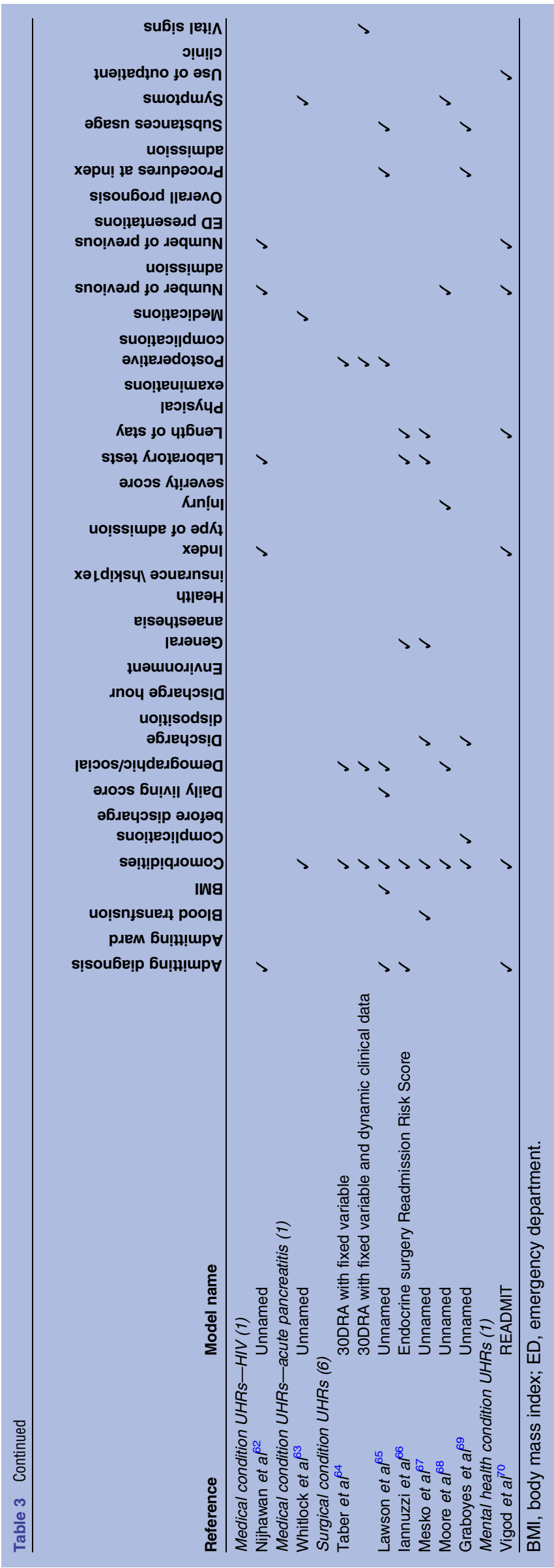

included studies were qualitatively synthesised and presented in narrative form.

\section{RESULTS}

\section{Literature search result}

The initial electronic database search produced 7310 records. After removal of 1798 duplicates, a total of 5512 references of potential relevance to this systematic review remained. Titles and abstracts were then appraised and excluded 5333 records due to irrelevance. Of the remaining 179 relevant references, 98 were excluded as they were conference abstracts. A total of 81 references were reviewed as full text and a further 21 were excluded against selection criteria. A total of 18 of the 21 excluded studies developed and/or validated risk predictive models for the 48 -hour ${ }^{73}$ or 72 -hour ${ }^{74}$ intensive care unit readmissions or the 3-month to 1-year unplanned hospital readmissions. ${ }^{75-90}$ One study focused on participants who were discharged to a hospital in the home-hospital programme receiving intravenous antibiotics. ${ }^{91}$ The other study, ${ }^{92}$ which had been included in the previous systematic review, ${ }^{10}$ was also excluded. It was also found that the same result was published in two articles; ${ }^{32}$ therefore, the later year article ${ }^{32}$ was excluded. A hand search of reference list of the remaining 60 articles was also conducted and no additional studies were identified. Finally, a total of 60 studies were included in this systematic review. Figure 1 is a flow chart as per the Preferred Reporting Items for Systematic Reviews and Meta-Analyses of the screening process of the database search results. The overall risk of bias of the 60 studies was low when evaluated against the six domains of potential bias. All studies described the population of interest adequately for key characteristics, the response rate information was clearly stated, adequate proportion of the study population had complete data on all independent variables, the outcome variable readmission was measured with sufficient accuracy and the method of statistical analysis was appropriate for the design of the study. ${ }^{72}$

\section{Study characteristics}

Table 1 summarises the characteristics of the final included studies of this systematic review. The 60 studies were conducted in several countries: USA $(n=41)$, Canada $(n=7)$, Australia $(n=3)$, Spain $(n=3)$, and one from Hong Kong, Korea, Israel, Italy, Singapore and the UK. Of the included studies, the majority employed retrospective data except two. One study ${ }^{18}$ used retrospective and prospective data and the other ${ }^{39}$ collected prospective data. Fifty-seven included studies accessed healthcare data of either tertiary hospital, centralised or national health information databases. The remaining three studies used community hospital data. ${ }^{19} 4454$ The duration of retrieved data source ranged from 1 single day across 10 hospitals $^{24}$ to 10 years ${ }^{47}$ of four healthcare databases. All included studies were based on adult 


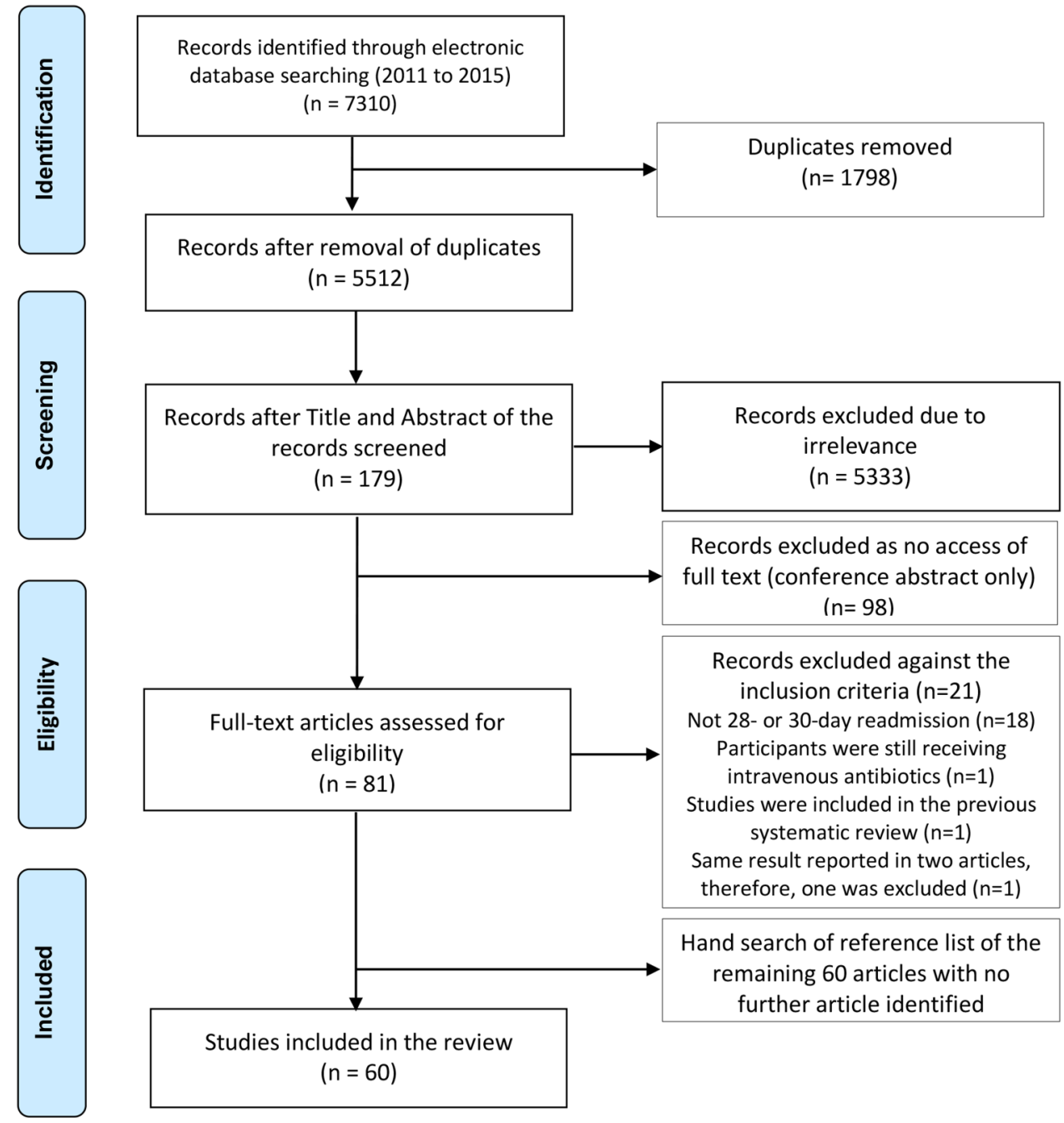

Figure 1 Flow chart for the search and study selection process (PRISMA). PRISMA, preferred reporting items for systematic reviews and meta-analyses.

patients' (aged $\geq 18$ years) healthcare data and the mean age, if reported, ranged from 43 to 85 years.

The 60 included studies reported unique 73 predictive models for 28-day or 30-day unplanned hospital readmissions. A total of 68 of the unique 73 predictive models were developed between 2011 and 2015 and 5 were existing models, which were further validated or applied to compare with other developed/existing models. The model utilisation outcome included all-cause admissions (14 studies), ${ }^{12}{ }^{13}{ }^{16-27}$ cardiovascular-related disease including pneumonia (24 studies, ${ }^{15}{ }^{28-50}$ of which 11 studies focused on heart failure only), medical/internal medicine conditions (15 studies), ${ }^{11} 1^{14}$ 51-63 surgical conditions (6 studies) ${ }^{64-69}$ and mental health conditions. ${ }^{70}$ A total of 17 models were based on administrative data and the remaining models were derived or validated using administrative and/or clinical/medical records data. The sample size varied from 100 patients ${ }^{46}$ to nearly a million ${ }^{57}$ patients. The unplanned hospital readmission rate ranged from $2.8 \%{ }^{66}(\mathrm{n}=34046)$ to $38 \%^{46}(\mathrm{n}=100)$.
Performance of predictive models for 28-day or 30-day unplanned hospital readmissions

Table 2 displays the measures of all included predictive models. Multivariable logistic regression model was used in all included studies. In logistic regression, the outcome variable is the log of the odds of the event (probability of readmission/(1-probability of readmission)). Once the final model is determined, the multivariable logistic regression allows for the calculation of probability of readmission for cohort studies. The predicted probabilities of the final multivariable logistic model are also used for computing the receiver operating characteristic (ROC) curve and the calculation of the ROC, a measure of model discrimination.

Overall, 56 of the 60 included studies reported model discriminative ability (C-statistic), ranging from $0.21^{46}$ to 0.88. ${ }^{63}$ The area under curve for validation studies ranged from $0.53^{30}$ to $0.83,{ }^{63}$ being slightly lower than those for the derivation study, $0.21^{46}$ to $0.88 .^{63}$ For allcause unplanned hospital readmission models, the C-statistic was reported by 14 studies ranging from $0.55^{13}$ 
to $0.80 .{ }^{22}$ Among 16 developed models and 2 existing models, 8 new models and 2 existing models had a C-statistic value $>0.70 .^{12} 16171922232627$

Regarding cardiovascular disease-related readmissions (24 studies), the C-statistic ranged from $0.21^{46}$ to $0.836^{32}$ across 32 developed models and 5 existing models. Of those, only nine developed models had a C-statistic value $>0.70 .^{30} 3234353840414950$ In particular, 13 of the 17 models (12 developed and 5 existing) from 11 studies with the special focus on heart failure-related readmissions were presented with C-statistic $<0.70 .{ }^{39} 4042-48$ For surgical-related readmissions (6 studies), the C-statistic ranged from $0.59^{67}$ to $0.85^{69}$ among 7 developed models. Three of the seven models showed moderate-to-high discrimination ability. ${ }^{64} 6569$ Patients with heart failure in the telemonitoring program were less likely to be admitted, with the reported C-statistic being $0.21{ }^{46}$ This indicates that the telemonitoring program was effective in identifying and intervening in patients who were reporting symptoms and thus reduced the likelihood of readmission.

However, 10 of 13 developed models and 1 existing model for medical condition-related readmissions (15 studies) were found to have consistent moderate discrimination ability. Four developed models also demonstrated high discrimination ability with C-statistic exceeding 0.80 .53525763

This updated systematic review also identified one study on mental health condition-related unplanned hospital readmission. A predictive model, READMIT $<$ (R) Repeat admissions; (E) Emergent admissions; (D) Diagnoses, and unplanned Discharge; (M) Medical comorbidity; (I) prior service use Intensity; and (T) Time in hospital>, was derived and validated using a 3-year Canadian National Health Database with a C-statistic of 0.63 .

One existing predictive model, the LACE index, although validated by eight studies, demonstrated inconsistent model performance. The LACE index was first developed by van Walraven $e t a t^{93}$ in 2010 to predict the risk of unplanned readmission or death within 30 days after hospital discharge in medical and surgical patients. The model was derived and validated based on administrative data with a C-statistic of 0.684. The model includes the length of hospitalisation stay (L), acuity of the admission (A), comorbidities of patients (C) and number of emergency department visits in the 6 months before admission (E). Five studies validated the LACE index model using healthcare data of Canada, Singapore, the UK and the USA to predict all-cause readmission (4), ${ }^{13} \quad 16 \quad 17 \quad 26$ heart failure readmission (1) ${ }^{44}$ and general medical condition-related readmission (2). ${ }^{58} 56$ The discriminative ability of the model (C-statistic), reported by six studies, varied from 0.51 to 0.72. ${ }^{13} 1617265658$

An extension of the LACE index to predict early death or all-cause 30-day urgent hospital readmission was further derived using administrative healthcare data and named as LACE+ index by van Walraven $e t a l^{27}$ in 2012. The LACE + index, in addition to four predictive variables, included patient age and sex, teaching status of the discharging hospital, acute diagnoses and procedures performed during the index admission, number of days on alternative level of care during the index admission and number of elective and urgent admissions to hospital in the year before the index admission. The LACE+ index had a C-statistic of 0.771, which exceeded the performance of LACE index. The LACE+ index was further validated by two large Canadian retrospective studies. The performance of the model was $0.61^{47}$ for patients with heart failure and $0.73^{23}$ for patients with all-cause hospital readmissions.

A Canadian study compared the performance of different models within the same population for 30-day readmission or death due to heart failure. A total of 59652 patients' admission information was retrieved from four health databases over a 10 -year period. Five models were examined in the study, ${ }^{47}$ namely Charlson, CMS Krumholz, Keenan, LACE index and LACE+. The five models had the C-statistic of 0.570.61 . In terms of types of data sources used to develop or validate the 73 unique predictive models, administrative healthcare data were used for 17 models but were found/identified with inconsistent discriminative ability. A total of 13 of the 17 models reported C-statistic between 0.55 and 0.7 , and the remaining four models reported C-statistic between 0.7 and 0.876 . Similarly, the performance of the remaining 56 models using clinical/medical data varied between 0.21 and 0.88 (C-statistic).

Only two models ${ }^{32} 53$ were developed targeting the potentially avoidable/preventable unplanned hospital readmissions. The outcome measure of the models focused on the end-of-life patients ${ }^{53}$ and pneumonia, heart failure, acute myocardial infarction and chronic obstructive pulmonary disease. ${ }^{32}$ Both models had C-statistic $>0.8$ (0.85 and 0.83 , respectively).

Sensitivity and specificity were calculated by 16 of the 60 included studies. The sensitivity of the predictive model ranged from 5.4\% (PARR-30 model, Patients at Risk of Re-admission within 30 days) ${ }^{11}$ to $91.95 \%$ (potentially avoidable readmission (PAR) model), ${ }^{32}$ while specificity values were between $22 \%$ (Rehospitalisation Risk Score) $^{24}$ and $99.5 \%$ (PARR-30 model). ${ }^{11}$

A total of 14 of the 60 included studies reported the PPV $\left(5.6^{41}-86.61 \%^{32}\right)$ and NPV $\left(19.1^{61}-99.1 \%^{41}\right)$ of the readmission risk predictive model. Similarly, only 17 studies calibrated the developed predictive models and mostly presented as $p$ value, except one study ${ }^{68}$ that reported the model calibration as the value of intercept and slope.

Predictive risk of readmission was assessed in all included studies, but only 14 of the included 60 studies specified thresholds for risk categories. Thresholds ranged from $4 \%{ }^{35}$ to $80 \%{ }^{61}$ 
Key variables included in the readmission risk predictive model

A total of 28 types of significant variables were extracted from the 73 unique predictive models for unplanned hospital readmissions as shown in table 3. Overall, the top 10 significant variables included in the 73 risk predictive models are comorbidities $(n=54)$, demographic/ social $(n=45)$, length of stay $(n=29)$, number of previous admissions $(n=29)$, laboratory tests $(n=25)$, medications $(n=21)$, index type of admission $(n=17)$, procedures at index admission $(n=16)$, admitting diagnosis $(n=14)$ and number of previous emergency department presentations $(\mathrm{n}=14)$ (refer to figure 2). The key demographic/ social variables consisted of age $(n=26)$, gender $(n=25)$, living arrangement $(\mathrm{n}=12)$, race $(\mathrm{n}=8)$ and marital status $(n=6)$.

The variables 'comorbidities', 'length of stay' and 'number of previous admissions' remained as the most frequently cited predictive risk variables against all utilisation outcomes. However, the variables 'laboratory tests' and 'medication' were more commonly included in the predictive models for cardiovascular disease-related and medical condition-related unplanned hospital readmissions compared with all-cause, mental health and surgical condition-related unplanned hospital readmissions.

\section{DISCUSSION}

A total of 60 studies with 73 unique risk predictive models for 28-day or 30-day unplanned hospital readmissions were included in this systematic review. The discrimination ability (C-statistic) of the 73 models varied largely from 0.21 to 0.88 . Inconsistent performances were found among models for all-cause readmission, cardiovascular disease-related readmission and surgical-related readmission. However, most of the predictive models for the general medical condition-related readmission exceeded C-statistic of 0.7. In comparison, Kansagara et $a l^{10}$ included 26 models with the focus of adult medical patients only. A total of 13 predictive models measured 30-day readmissions; of these, 10 models performed poorly and only 3 models reported C-statistic $>0.70$. The outcome measures of the other 13 models ranged from 41-day to 4-year unplanned hospital readmission; as a result of the vast difference in the time frame, the C-statistic also varied from 0.53 to 0.75 .

This updated systematic review has certain limitations. The studies included in this systematic review were limited compared with studies that were published in English with full-text access. The outcomes of the predictive models included in this systematic review were also restricted to 28-day or 30-day unplanned hospital readmission. A meta-analysis is not permitted in this systematic review as the included studies were heterogeneous due to diversity of cohort of population, duration of retrieved data source, sample sizes and geographical locations. It was noted that the sample size was reported in different units, that is, (index) admission/hospitalisation, cases, patients or discharges, as shown in table 1. The lack of standardised calculation could also

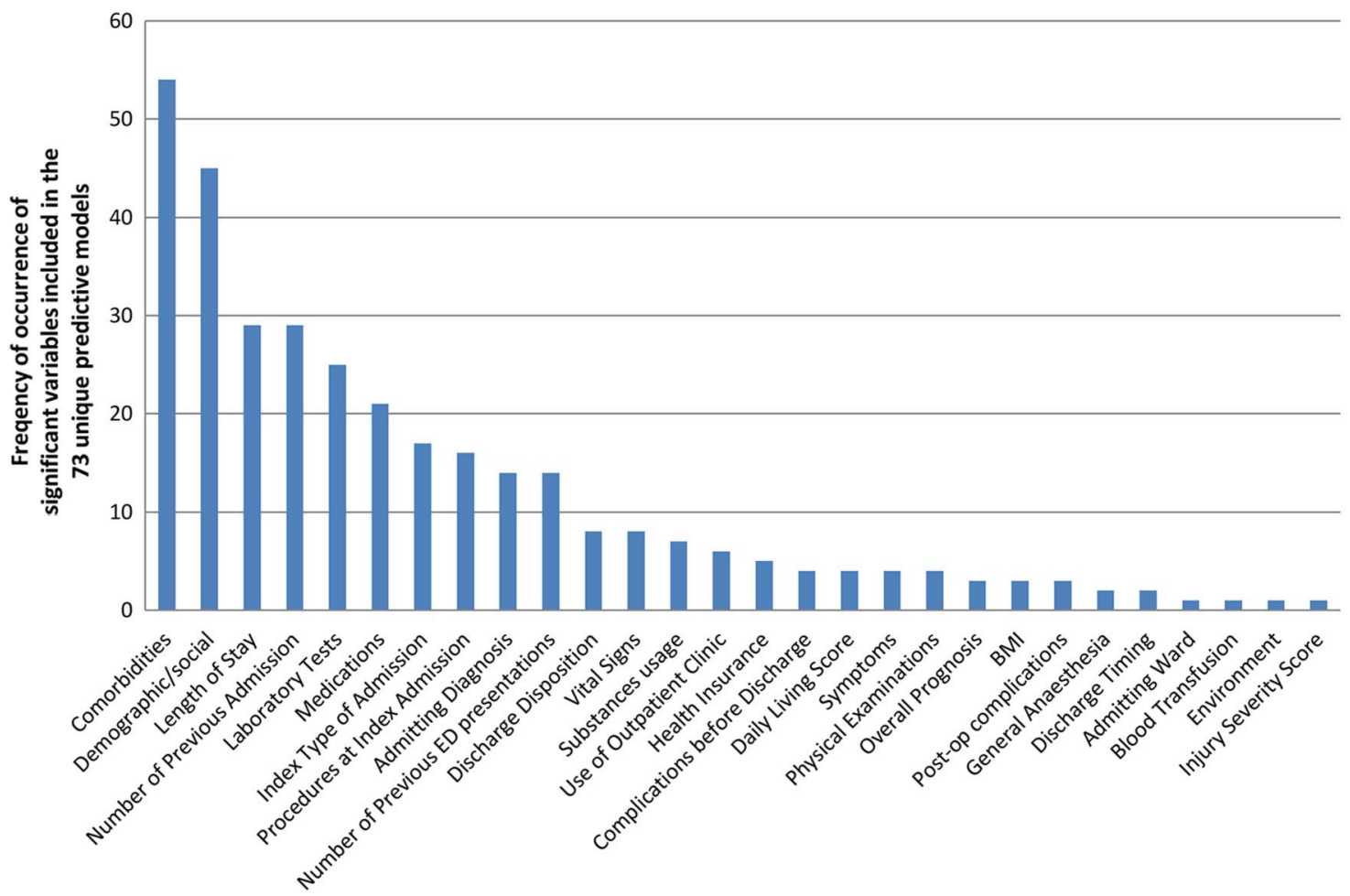

Figure 2 Pareto chart of significant variables included in the predictive models. BMI, body mass index; ED, emergency department. 
contribute to the broad range of readmission rates $(2.8-$ $38 \%$ ); thus, the results were not comparable. This systematic review also found the sample size is not associated with the model predictive ability. Of the included 73 unique models, Zai et $a l^{46}$ derived a model based on the selected 100 readmitted patients with heart failure and scored the lowest C-statistic of 0.21. In contrast, Whitlock $e t a l^{63}$ retrieved around 200 readmitted patients with acute pancreatitis and developed a model with the highest discrimination ability ( $\mathrm{C}$-statistic $=0.88)$.

There has been increased recognition that some unplanned hospital readmissions are associated with the diagnosis of the initial hospitalisation and could be potentially prevented or avoided through systematic discharge process. In 2006, a Swiss study ${ }^{94}$ compared three models (non-clinical model, Charlson-based model and SQLape model, A patient classification system, also designed to adjust for costs and other outcomes) to identify potentially preventable readmission risk on over 60000 medical patients. The C-statistics of the three models were $0.67,0.69$ and 0.72 , respectively, which indicated poor-to-reasonable discrimination ability. In contrast, this systematic review identified two highperformance models ${ }^{32} 53$ for potentially avoidable/preventable readmissions with $\mathrm{C}$-statistic $>0.8$. The PAR model $^{32}$ was also high in other predictive model performance indicators, such as sensitivity $(91.95 \%)$, specificity $(97.65 \%)$, PPV (86.61\%) and NPV (98.65\%). However, the two models were developed based on comparatively smaller sample size of $5600^{32}$ and $10275^{53}$ using American healthcare data collected over a 12-month period. Overall, the number of potentially preventable readmissions remains unclear due to lack of standardised identification process. ${ }^{95-98}$

Compared with the previous systematic review, ${ }^{10}$ there were more studies in this review using clinical medical record data to develop disease-specific predictive models. However, the debate whether a predictive model should be developed using administrative data or clinical/ medical records data remains inconclusive. Three key variables extracted from the 73 unique models, 'comorbidity', 'length of stay' and 'previous admissions', were based on administrative data and were consistent with the findings of a previous systematic review. ${ }^{10}$ The latest evidence has shown that variables based on clinical medical data, that is, 'laboratory tests' and 'medications', were also valued in models for predicting cardiovascular-related and medical condition-related readmissions. Of note, ineffective communication in transitions of care is reported as a major contributing factor to adverse events that directly risk patient safety ${ }^{99}{ }^{100}$ Poor communication at discharge also leads to preventable unplanned readmissions and frequent problems with the continuity of medication management. ${ }^{101-103}$ None of the examined 73 models cited the comprehensiveness of discharge information as a predictor to unplanned hospital readmissions.

All included studies in this systematic review were based on adult population. To date, only two paediatric predictive models were identified and both were based on American paediatric populations. One retrospective multicentre study $^{104}$ retrieved 12 -month administrative data from 38 children's hospitals. A model was developed and internally validated with a high discrimination ability (C-statistic $=0.81$ ). However, the model outcome measure was 12-month all-cause readmissions. In comparison, a 30-day hospital readmission model ${ }^{105}$ was developed based on 5376 paediatric patients following plastic surgery procedures. The study accessed prospective medical records, and the model had moderate discrimination ability of C-statistic 0.784 .

The performance of the 73 unique predictive models in this review was assessed using a variety of statistical measures. Inconsistency of reported statistical measures was noted in the included 60 studies, of which 2 studies $^{44} 58$ reported threshold as the only model performance measurement. A US framework for assessing the performance of predictive models ${ }^{106}$ argued the importance of reporting discrimination and calibration for a risk predictive model. In all included 60 studies, the most reported measure of the risk predictive model is the ROC (C-statistic). The interpretation of the risk predictive model discriminative ability (C-statistic) was inconsistent. For instance, a study ${ }^{47}$ examined five predictive models and concluded that the models had moderate discrimination ability based on the C-statistic of $0.57-0.6$; whereas models are typically considered reasonable when the C-statistic is higher than 0.7 by Hosmer and Lemeshow. ${ }^{71}$

\section{CONCLUSION}

The risk predictive models which focused on general medical conditions in relation to unplanned hospital readmissions reported moderate discriminative ability. Two models ${ }^{32}{ }^{53}$ for potentially preventable/avoidable readmissions showed high discriminative ability. This systematic review, however, found inconsistent performance across the included unique 73 risk predictive models for unplanned hospital readmissions.

The variables 'comorbidities', 'length of stay' and 'previous admissions' were frequently cited across the examined unique 73 models, and 'laboratory tests' and 'medication' variables had more weight in the models for cardiovascular disease and medical conditions in relation to readmissions. However, comprehensiveness of discharge information was not included in any of the examined models.

This review highlighted the need for rigorous validation of the risk predictive models with moderate-to-high discriminative ability be undertaken, especially the two models $^{32} 53$ for the potentially avoidable hospital readmissions. There is a need to review and update predictive models. Specifically this is essential for paediatric 28-day all-cause unplanned hospital readmissions as limited evidence was found.

Findings from this updated systematic review revealed an increasing number of developed risk predictive 
models for specific disease-related unplanned hospital readmission using clinical/medical records data. Findings from this systematic review also confirm the limited applicability of hospital readmission risk predictive models. The performance of the applied existing models was inconsistent. It is, therefore, essential to clearly define utilisation outcomes and the type of accessible data sources prior to determining which risk predictive model to use. For example, most of the models were developed based on healthcare data from the USA, which might not be applicable to patients from other settings.

Acknowledgements The authors would like to acknowledge Ms Marta Rossignoli, librarian of Child and Adolescent Health Service, Western Australia, for her assistance in the literature search.

Contributors $\mathrm{HZ}, \mathrm{SD}$ and PD contributed to the design of the review. $\mathrm{HZ}, \mathrm{SD}$, PD and $L G$ acquired the data. HZ, SD, PD, PR and LG contributed to analysis. $\mathrm{HZ}$ wrote the draft. $\mathrm{HZ}, \mathrm{SD}, \mathrm{PR}$ and $\mathrm{PD}$ critically revised the intellectual content of this work.

Funding This study is funded by a grant from the Australian Research Council-ARC Linkage Grant (Project ID: LP140100563). HZ is also supported by the Academic Research Grant from the Nursing and Midwifery Office, Western Australia Department of Health.

Competing interests None declared.

Provenance and peer review Not commissioned; externally peer reviewed.

Data sharing statement No additional data are available.

Open Access This is an Open Access article distributed in accordance with the Creative Commons Attribution Non Commercial (CC BY-NC 4.0) license, which permits others to distribute, remix, adapt, build upon this work noncommercially, and license their derivative works on different terms, provided the original work is properly cited and the use is non-commercial. See: http:// creativecommons.org/licenses/by-nc/4.0/

\section{REFERENCES}

1. Bosco JA III, Karkenny AJ, Hutzler LH, et al. Cost burden of 30-day readmissions following Medicare total hip and knee arthroplasty. $J$ Arthroplasty 2014;29:903-5.

2. Joynt KE, Jha AK. Thirty-day readmissions-truth and consequences. N Engl J Med 2012;366:1366-9.

3. Jencks SF, Williams MV, Coleman EA. Rehospitalizations among patients in the Medicare fee-for-service program. N Engl J Med 2009;360:1418-28.

4. Centers for Medicare \& Medicaid Services. Table 5.1b. Discharges, Total Days of Care, Total Charges, and Program Payments for Medicare Beneficiaries Discharged from Short-Stay Hospitals, by Type of Entitlement: Calendar Years 1972-2006. 2007: https:// www.cms.gov/Research-Statistics-Data-and-Systems/StatisticsTrends-and-Reports/MedicareMedicaidStatSupp/2007.html

5. Health \& Social Care Information Centre. Hospital Episode Statistics, Admitted Patient Care-England, 2010-11 [NS]. 2011: http://www.hscic.gov.uk/pubs/hesadmitted1011

6. Stefan MS, Pekow PS, Nsa W, et al. Hospital performance measures and 30-day readmission rates. J Gen Intern Med 2013:28:377-85

7. Wish JB. The role of 30-day readmission as a measure of quality. Clin J Am Soc Nephrol 2014;9:440-2.

8. U.S. Department of Health \& Human Services. Readmissions to hospital: percentage of unplanned readmissions within 28 days of separation, during the 6-month time period. 2012. http://www. qualitymeasures.ahrq.gov.

9. Australian Institute of Health and Welfare. National healthcare agreement: PI23-Unplanned hospital readmission rates. 2013. http://meteor.aihw.gov.au/content/index.phtml/itemld/497129

10. Kansagara $D$, Englander $\mathrm{H}$, Salanitro $A$, et al. Risk prediction models for hospital readmission: a systematic review. JAMA 2011;306:1688-98.
11. Billings J, Blunt I, Steventon A, et al. Development of a predictive model to identify inpatients at risk of re-admission within 30 days of discharge (PARR-30). BMJ Open 2012;2:pii: e001667.

12. Choudhry SA, Li J, Davis D, et al. A public-private partnership develops and externally validates a 30-day hospital readmission risk prediction model. Online J Public Health Inform 2013;5:219.

13. Cotter PE, Bhalla VK, Wallis SJ, et al. Predicting readmissions: poor performance of the LACE index in an older UK population. Age Ageing 2012;41:784-9.

14. Donzé J, Aujesky D, Williams D, et al. Potentially avoidable 30-day hospital readmissions in medical patients: derivation and validation of a prediction model. JAMA Intern Med 2013;173:632-8.

15. Hebert C, Shivade C, Foraker R, et al. Diagnosis-specific readmission risk prediction using electronic health data: a retrospective cohort study. BMC Med Inform Decis Mak 2014;14:65.

16. Escobar GJ, Ragins A, Scheirer P, et al. Nonelective rehospitalizations and postdischarge mortality: predictive models suitable for use in real time. Med Care 2015;53:916-23.

17. Yu S, Farooq $F$, van Esbroeck $A$, et al. Predicting readmission risk with institution-specific prediction models. Artif Intell Med 2015;65:89-96.

18. Baillie CA, VanZandbergen $\mathrm{C}$, Tait $\mathrm{G}$, et al. The readmission risk flag: using the electronic health record to automatically identify patients at risk for 30-day readmission. $J$ Hosp Med 2013;8:689-95.

19. Gildersleeve R, Cooper P. Development of an automated, real time surveillance tool for predicting readmissions at a community hospital. Appl Clin Inform 2013;4:153-69.

20. Kruse RL, Hays HD, Madsen RW, et al. Risk factors for all-cause hospital readmission within 30 days of hospital discharge. J Clin Outcomes Manage 2013;20:203-14.

21. Richmond DM. Socioeconomic predictors of 30-day hospital readmission of elderly patients with initial discharge destination of home health care. University of Alabama at Birmingham, 2013.

22. Shulan M, Gao K, Moore CD. Predicting 30-day all-cause hospital readmissions. Health Care Manag Sci 2013;16:167-75.

23. van Walraven $\mathrm{C}$, Wong J, Forster AJ, et al. Predicting post-discharge death or readmission: deterioration of model performance in population having multiple admissions per patient. J Eval Clin Pract 2013;19:1012-18.

24. Khan A, Malone ML, Pagel P, et al. An electronic medical record-derived real-time assessment scale for hospital readmission in the elderly. WMJ 2012;111:119-23.

25. Lee EW. Selecting the best prediction model for readmission. $J$ Prev Med Public Health 2012;45:259-66.

26. van Walraven C, Wong J, Forster AJ. Derivation and validation of a diagnostic score based on case-mix groups to predict 30-day death or urgent readmission. Open Med 2012;6:e90-100.

27. van Walraven $\mathrm{C}$, Wong J, Forster AJ. LACE+ index: extension of a validated index to predict early death or urgent readmission after hospital discharge using administrative data. Open Med 2012;6: e80-90.

28. Iannuzzi JC, Chandra A, Kelly KN, et al. Risk score for unplanned vascular readmissions. J Vasc Surg 2014;59:1340-47.e1.

29. Keyhani S, Myers LJ, Cheng E, et al. Effect of clinical and social risk factors on hospital profiling for stroke readmission: a cohort study. Ann Intern Med 2014;161:775-84.

30. Rana S, Tran T, Wei L, et al. Predicting unplanned readmission after myocardial infarction from routinely collected administrative hospital data. Aust Health Rev 2014;38:377-82.

31. Shahian DM, He X, O'Brien S, et al. Development of a clinical registry-based 30-day readmission measure for coronary artery bypass grafting surgery. Circulation 2014;130:399-409.

32. Shams I, Ajorlou S, Yang K. A predictive analytics approach to reducing 30-day avoidable readmissions among patients with heart failure, acute myocardial infarction, pneumonia, or COPD. Health Care Manag Sci 2015;18:19-34.

33. Sharif R, Parekh TM, Pierson KS, et al. Predictors of early readmission among patients 40 to 64 years of age hospitalized for chronic obstructive pulmonary disease. Ann Am Thorac Soc 2014;11:685-94.

34. Lucas DJ, Haider A, Haut E, et al. Assessing readmission after general, vascular, and thoracic surgery using ACS-NSQIP. Ann Surg 2013;258:430-9.

35. Wallmann R, Llorca J, Gomez-Acebo I, et al. Prediction of 30-day cardiac-related-emergency-readmissions using simple administrative hospital data. Int J Cardiol 2013:164:193-200.

36. Wasfy JH, Rosenfield K, Zelevinsky K, et al. A Prediction model to identify patients at high risk for 30-day readmission after percutaneous coronary intervention. Circ Cardiovasc Qual Outcomes 2013;6:429-35. 
37. Krumholz HM, Lin Z, Drye EE, et al. An administrative claims measure suitable for profiling hospital performance based on 30-day all-cause readmission rates among patients with acute myocardial infarction. Circ Cardiovasc Qual Outcomes 2011;4:243-52.

38. Betihavas V, Frost SA, Newton PJ, et al. An absolute risk prediction model to determine unplanned cardiovascular readmissions for adults with chronic heart failure. Heart Lung Circ 2015;24:1068-73.

39. Di Tano G, De Maria R, Gonzini L, et al. The 30-day metric in acute heart failure revisited: data from IN-HF Outcome, an Italian nationwide cardiology registry. Eur J Heart Fail 2015;17:1032-41.

40. Huynh QL, Saito M, Blizzard CL, et al. Roles of nonclinical and clinical data in prediction of 30-day rehospitalization or death among heart failure patients. J Card Fail 2015;21:374-81.

41. Raposeiras-Roubin S, Abu-Assi E, Cambeiro-Gonzalez C, et al Mortality and cardiovascular morbidity within 30 days of discharge following acute coronary syndrome in a contemporary European cohort of patients: how can early risk prediction be improved? The six-month GRACE risk score. Revista Portuguesa de Cardiologia 2015;34:383-91.

42. Sudhakar S, Zhang W, Kuo YF, et al. Validation of the readmission risk score in heart failure patients at a tertiary hospital. $J$ Card Fail 2015;21:885-91.

43. Fleming LM, Gavin M, Piatkowski G, et al. Derivation and validation of a 30-day heart failure readmission model. Am J Cardiol 2014;114:1379-82.

44. Wang $\mathrm{H}$, Robinson RD, Johnson $\mathrm{C}$, et al. Using the LACE index to predict hospital readmissions in congestive heart failure patients. BMC Cardiovasc Disord 2014;14:97.

45. Eapen ZJ, Liang L, Fonarow GC, et al. Validated, electronic health record deployable prediction models for assessing patient risk of 30-day rehospitalization and mortality in older heart failure patients. JACC Heart Fail 2013;1:245-51.

46. Zai AH, Ronquillo JG, Nieves R, et al. Assessing hospital readmission risk factors in heart failure patients enrolled in a telemonitoring program. Int J Telemed Appl 2013;2013:305819.

47. Au AG, McAlister FA, Bakal JA, et al. Predicting the risk of unplanned readmission or death within 30 days of discharge after a heart failure hospitalization. Am Heart J 2012;164:365-72.

48. Watson AJ, O'Rourke J, Jethwani K, et al. Linking electronic health record-extracted psychosocial data in real-time to risk of readmission for heart failure. Psychosomatics 2011;52:319-27.

49. Mather JF, Fortunato GJ, Ash JL, et al. Prediction of pneumonia 30-day readmissions: a single-center attempt to increase model performance. Respir Care 2014;59:199-208.

50. Lindenauer PK, Normand SL, Drye EE, et al. Development validation, and results of a measure of 30-day readmission following hospitalization for pneumonia. J Hosp Med 2011;6:142-50.

51. Shadmi E, Flaks-Manov N, Hoshen M, et al. Predicting 30-day readmissions with preadmission electronic health record data. Med Care 2015;53:283-9.

52. Tsui E, Au SY, Wong CP, et al. Development of an automated model to predict the risk of elderly emergency medical admissions within a month following an index hospital visit: a Hong Kong experience. Health Inform J 2015;21:46-56.

53. Donze J, Lipsitz S, Schnipper JL. Risk factors for potentially avoidable readmissions due to end-of-life care issues. J Hosp Med 2014;9:310-14.

54. He D, Mathews SC, Kalloo AN, et al. Mining high-dimensional administrative claims data to predict early hospital readmissions. J Am Med Inform Assoc 2014;21:272-9.

55. Taha M, Pal A, Mahnken JD, et al. Derivation and validation of a formula to estimate risk for 30 -day readmission in medical patients. Int J Qual Health Care 2014;26:271-7.

56. Tan SY, Low LL, Yang Y, et al. Applicability of a previously validated readmission predictive index in medical patients in Singapore: a retrospective study. BMC Health Serv Res 2013;13:366.

57. Zapatero A, Barba R, Marco J, et al. Predictive model of readmission to internal medicine wards. Eur J Intern Med 2012;23:451-6.

58. Gruneir A, Dhalla IA, van Walraven C, et al. Unplanned readmissions after hospital discharge among patients identified as being at high risk for readmission using a validated predictive algorithm. Open Med 2011;5:e104-e11.

59. Singal AG, Rahimi RS, Clark C, et al. An automated model using electronic medical record data identifies patients with cirrhosis at high risk for readmission. Clin Gastroenterol Hepatol 2013;11:1335-41.e1.
60. Volk ML, Tocco RS, Bazick J, et al. Hospital re-admissions among patients with decompensated cirrhosis. Am J Gastroenterol 2012;107:247-52

61. Perkins RM, Rahman A, Bucaloiu ID, et al. Readmission after hospitalization for heart failure among patients with chronic kidney disease: a prediction model. Clin Nephrol 2013;80:433-40.

62. Nijhawan AE, Clark C, Kaplan R, et al. An electronic medical record-based model to predict 30-day risk of readmission and death among HIV-infected inpatients. J Acquir Immune Defic Syndr 2012;61:349-58.

63. Whitlock TL, Tignor A, Webster EM, et al. A scoring system to predict readmission of patients with acute pancreatitis to the hospital within thirty days of discharge. Clin Gastroenterol Hepatol 2011;9:175-80; quiz e18.

64. Taber DJ, Palanisamy AP, Srinivas TR, et al. Inclusion of dynamic clinical data improves the predictive performance of a 30-day readmission risk model in kidney transplantation. Transplantation 2015;99:324-30.

65. Lawson EH, Hall BL, Louie R, et al. Identification of modifiable factors for reducing readmission after colectomy: a national analysis. Surgery 2014;155:754-66.

66. Iannuzzi JC, Fleming FJ, Kelly KN, et al. Risk scoring can predict readmission after endocrine surgery. Surgery 2014;156:1432-38; discussion 1438-40.

67. Mesko NW, Bachmann KR, Kovacevic D, et al. Thirty-day readmission following total hip and knee arthroplasty: a preliminary single institution predictive model. J Arthroplasty 2014;29:1532-8.

68. Moore L, Stelfox HT, Turgeon AF, et al. Derivation and validation of a quality indicator for 30-day unplanned hospital readmission to evaluate trauma care. J Trauma Acute Care Surg 2014;76:1310-16.

69. Graboyes EM, Liou TN, Kallogjeri D, et al. Risk factors for unplanned hospital readmission in otolaryngology patients. Otolaryngol Head Neck Surg 2013;149:562-71.

70. Vigod SN, Kurdyak PA, Seitz D, et al. READMIT: a clinical risk index to predict 30-day readmission after discharge from acute psychiatric units. J Psychiatr Res 2015;61:205-13.

71. Hosmer DW, Lemeshow S. Applied logistic regression. John Wiley and Sons: New York, 2000.

72. Hayden JA, Cote P, Bombardier C. Evaluation of the quality of prognosis studies in systematic reviews. Ann Intern Med 2006;144:427-37.

73. Brown SE, Ratcliffe SJ, Kahn JM, et al. The epidemiology of intensive care unit readmissions in the United States. Am J Respir Crit Care Med 2012;185:955-64.

74. Abu-Awwad R, Buran G. Predictors of early readmission to the intensive care unit. Chest 2012;142:280A.

75. Weidmann ZM, Breidthardt T, Twerenbold R, et al. Prediction of mortality using quantification of renal function in acute heart failure. Int J Cardiol 2015;201:650-7.

76. $\mathrm{Yu} X$, Sun $\mathrm{Y}$, Zhao $\mathrm{Y}$, et al. Prognostic value of plasma galectin-3 levels in patients with coronary heart disease and chronic heart failure. Int Heart J 2015;56:314-18.

77. Alassaad A, Melhus H, Hammarlund-Udenaes M, et al. A tool for prediction of risk of rehospitalisation and mortality in the hospitalised elderly: secondary analysis of clinical trial data. BMJ Open 2015;5:e007259.

78. Ambler GK, Brooks DE, Al Zuhir N, et al. Effect of frailty on shortand mid-term outcomes in vascular surgical patients. Br J Surg 2015;102:638-45

79. Bohlen Delgado AP, Marmelo B, Abreu L, et al. A score of risk of events in elderly patients with acute myocardial infarction without ST elevation. Eur J Heart Fail 2015;17:345.

80. Brudvik KW, Mise Y, Conrad C, et al. Definition of readmission in 3,041 patients undergoing hepatectomy. J Am Coll Surg 2015;221:38-46

81. Cui Y, Metge C, Ye X, et al. Development and validation of a predictive model for all-cause hospital readmissions in Winnipeg, Canada. J Health Serv Res Policy 2015;20:83-91.

82. Fry DE, Pine M, Locke D, et al. Composite measurement of outcomes in Medicare inpatient laparoscopic cholecystectomy. J Am Coll Surg 2015;221:102-9.

83. Sciatti E, Vizzardi E, Bonadei I, et al. Prognostic value of RV isovolumic acceleration and tissue strain in moderate HFrEF. Eur $J$ Clin Invest 2015;45:1052-9.

84. Shirakabe A, Hata N, Kobayashi N, et al. Serum heart-type fatty acid-binding protein level can be used to detect acute kidney injury on admission and predict an adverse outcome in patients with acute heart failure. Circ $J$ 2015;79:119-28.

85. Sinning JM, Wollert KC, Sedaghat A, et al. Risk scores and biomarkers for the prediction of 1-year outcome after transcatheter aortic valve replacement. Am Heart J 2015;170:821-9. 
86. Sur MD, Namm JP, Hemmerich JA, et al. Radiographic sarcopenia and self-reported exhaustion independently predict NSQIP serious complications after pancreaticoduodenectomy in older adults. Ann Surg Oncol 2015;22:3897-904.

87. Takahashi T, Kumamaru M, Jenkins S, et al. In-patient step count predicts re-hospitalization after cardiac surgery. $J$ Cardiol 2015;66:286-91.

88. Ben-Chetrit E, Chen-Shuali C, Zimran E, et al. A simplified scoring tool for prediction of readmission in elderly patients hospitalized in internal medicine departments. Isr Med Assoc J 2012;14:752-6.

89. Hasin T, Marmor Y, Kremers W, et al. Readmissions after implantation of axial flow left ventricular assist device. J Am Coll Cardiol 2013;61:153-63.

90. Lemke KW, Weiner JP, Clark JM. Development and validation of a model for predicting inpatient hospitalization. Med Care 2012;50:131-9.

91. Allison GM, Muldoon EG, Kent DM, et al. Prediction model for 30-day hospital readmissions among patients discharged receiving outpatient parenteral antibiotic therapy. Clin Infect Dis 2014;58:812-19.

92. Allaudeen N, Schnipper J, Orav EJ, et al. Inability of providers to predict unplanned readmissions. J Gen Intern Med 2011;26:771-6.

93. Van Walraven C, Dhalla IA, Bell C, et al. Derivation and validation of an index to predict early death or unplanned readmission after discharge from hospital to the community. CMAJ 2010;182:551-7.

94. Halfon P, Eggli Y, Prêtre-Rohrbach I, et al. Validation of the potentially avoidable hospital readmission rate as a routine indicator of the quality of hospital care. Med Care 2006;44:972-81.

95. Halfon P, Eggli, $Y$, van Melle G, et al. Measuring potentially avoidable hospital readmissions. J Clin Epidemiol 2002;55:573-87.

96. Hechenbleikner EM, Makary MA, Samarov DV, et al. Hospital readmission by method of data collection. J Am Coll Surg 2013;216:1150-8.
97. Van Walraven C, Bennett C, Jennings A, et al. Proportion of hospital readmissions deemed avoidable: a systematic review. CMAJ 2011;183:e391-402.

98. van Walraven C, Jennings A, Forster AJ. A meta-analysis of hospital 30-day avoidable readmission rates. J Eval Clin Pract 2012;18:1211-18.

99. Aase K, Laugaland KA, Dyrstad DN, et al. Quality and safety in transitional care of the elderly: the study protocol of a case study research design (phase 1). BMJ Open 2013;3:pii: e003506.

100. Garvey KC, Wolpert HA, Rhodes ET, et al. Health care transition in patients with Type 1 diabetes. Diabetes Care 2012;35:1716-22.

101. Della P, Michael R. Evaluating global trends in clinical communication. ACORN 2012;25:19-20.

102. Rothwell M, Jukka C, Lum E, et al. Retrospective analysis of emergency readmissions to rural and regional hospitals. J Pharm Pract Res 2011;41:413-20.

103. Russell L, Doggett J, Dawda P, et al. Patient safety-handover of care between primary and acute care. 2011. http://leadclinicians. health.gov.au/internet/lcg/publishing.nsf/Content/ OBC80B14F241017BCA257A590021F2E8/\$File/Transitions\%20of \%20Care\%20report\%20FINAL\%207May13.pdf

104. Feudtner C, Levin JE, Srivastava R, et al. How well can hospital readmission be predicted in a cohort of hospitalized children? A retrospective, multicenter study. Pediatrics 2009:123:286-93

105. Tahiri Y, Fischer JP, Wink JD, et al. Analysis of risk factors associated with 30-day readmissions following pediatric plastic surgery: a review of 5376 procedures. Plast Reconstr Surg 2015;135:521-9.

106. Steyerberg EW, Vickers AJ, Cook NR, et al. Assessing the performance of prediction models: a framework for some traditional and novel measures. Epidemiology 2010;21:128-38. 